\title{
On the Riemann zeta-function I
}

\section{Masako Izumi and Shin-ichi Izumi}

\author{
Dedicated to Professor Bernhard H. Neumann
}

We prove an approximation formula for the Riemann zeta function. We show that a classical theorem:

$$
\zeta(s)=O\left(t^{(\lambda-\sigma) / 2}\right) \text { as } t \rightarrow \infty \quad(s=\sigma+i t)
$$

uniformly in the domain $\frac{3}{2} \leq \sigma<1$, is an immediate consequence of our approximation formula. Our method is real and free from complex analysis.

\section{Introduction and theorems}

1.1. The Riemann zeta function is defined by

$$
\zeta(s)=\sum_{n=1}^{\infty} 1 / n^{s} \quad(s=\sigma+i t)
$$

which converges in the half plane $\sigma>1$ and represents a regular function. Riemann proved that it is analytically continued to the whole plane and regular there except at the point $s=1$, which is its simple pole. Riemann supposed that $\zeta(s) \neq 0$ in the strip $\frac{z}{2}<\sigma<1$. This is called the Riemann hypothesis. Lindelöf conjectured that

$$
\zeta(s)=O\left(t^{\varepsilon}\right) \text { uniformly for } \frac{7}{2} \leq \sigma<1
$$

for any $\varepsilon>0$, which is equivalent to $\zeta\left(\frac{1}{2}+i t\right)=O\left(t^{\varepsilon}\right)$.

Received 21 April 1976. The authors express their cordial thanks to Professor Kurt Mahler for his kind advice and encouragement. 
We shall prove an approximation formula for the zeta function. THEOREM 1 .

$$
\zeta(s)=\sum_{j=1}^{t / 3 \pi} \int_{3 / 2}^{t / \pi j} \frac{e^{-i(t \log k-2 \pi j k)}}{k^{\sigma}} d k+o(\log t) \text { as } t \rightarrow \infty,
$$

uniformly in the interval $\leq 0<1$.

As an immediate consequence, we get

\section{THEOREM 2.}

$$
\zeta(s)=O\left(t^{(1-\sigma) / 2}\right) \text { as } t \rightarrow \infty
$$

uniformly in $\frac{1}{2} \leq 0<1$.

1.2. We use the notation $\sum_{n=a}^{b}=\sum_{n=[a]}^{[b]}$ and $k, \imath, m, n, \ldots$ are used to represent continuous variables as well as discrete variables.

\section{Proof of Theorem 1}

It is known that

$$
\zeta(s)=s \int_{1}^{\infty} \frac{J(u)}{u^{s+1}} d u+\frac{1}{s-1}+\frac{1}{2} \quad(R s>0)
$$

(see [2], p. 14), where

$$
J(u)=[u]-u+\frac{z}{2} \sim \sum_{n=1}^{\infty} \frac{\sin 2 \pi n u}{\pi u} .
$$

We suppose that $s=\sigma+i t, \frac{1}{2}<\sigma<1$, and $t$ is a large positive number. Then

$$
\begin{aligned}
\zeta(s) & =(\sigma+i t) \int_{1}^{\infty} \frac{J(u)}{u^{(1+\sigma)+i t}} d u+o(1) \\
& =i t \int_{1}^{\infty} \frac{J(u)}{u^{1+\sigma}} e^{-i \cdot t \log u} d u+o(1) \\
& =t \int_{1}^{\infty} \frac{J(u)}{u^{1+\sigma}}(i \cos (t \log u)+\sin (t \log u)) d u+o(1) \\
& =i P+P^{*}+O(1) .
\end{aligned}
$$


We shall estimate $P$ only since $P^{*}$ can be done quite similarly. Using the Fourier expansion of $J(u)$,

$$
\begin{aligned}
P & =\sum_{m=1}^{\infty} \frac{t}{\pi m} \int_{1}^{\infty} \cos (t \log u) \sin 2 \pi m u \frac{d u}{u^{\sigma+1}} \\
& =\int_{\frac{I}{2}}^{\infty} \frac{t}{\pi m}(d m+d J(m)) \int_{1}^{\infty} \cos (t \log u) \sin 2 \pi m u \frac{d u}{u^{\sigma+1}} \\
& =\left(P_{1}+P_{2}\right) / \pi .
\end{aligned}
$$

By the transformation $m u=v$,

$$
\begin{aligned}
P_{1} & =t \int_{\frac{1}{2}}^{\infty} \frac{d m}{m^{1-\sigma}} \int_{m}^{\infty} \cos \left(t \log \frac{v}{m}\right) \sin 2 \pi v \frac{d v}{v^{\sigma+1}} \\
& =t \int_{\frac{1}{2}}^{\infty} \frac{\sin 2 \pi v}{v^{\sigma+1}} d v \int_{\frac{1}{2}}^{v} \cos \left(t \log \frac{v}{m}\right) \frac{d m}{m^{1-\sigma}} .
\end{aligned}
$$

Further we write $t \log (v / m)=n$; then

$$
m=v e^{-n / t}, d m=-(v / t) e^{-n / t} d n,
$$

and then

$$
\begin{aligned}
P_{1} & =\int_{\frac{1}{2}}^{\infty} \frac{\sin 2 \pi v}{v} d v \int_{0}^{t \log 2 v} \frac{\cos n}{e^{\sigma n / t}} d n \\
& =\int_{\frac{1}{2}}^{\infty} d v\left(\int_{0}^{\infty} d n-\int_{t \log 2 v}^{\infty} d n\right)=O(1) .
\end{aligned}
$$

By integration by parts,

$$
\begin{aligned}
P_{2} & =t \int_{\frac{z}{2}}^{\infty} \frac{J(m)}{m^{2}} d m \int_{1}^{\infty} \cos (t \log u) \sin 2 \pi m u \frac{d u}{u^{\sigma+1}} \\
& \quad-2 \pi t \int_{\frac{z}{2}}^{\infty} \frac{J(m)}{m} d m \int_{1}^{\infty} \cos (t \log u) \cos 2 \pi m u \frac{d u}{u^{\sigma}} \\
& =Q_{1}-2 \pi Q_{2} .
\end{aligned}
$$

\section{Estimation of $Q_{1}$}

By changing the order of integration, 


$$
Q_{1}=t \int_{1}^{\infty} \frac{\cos (t \log u)}{u^{\sigma+1}} d u \int_{\frac{z_{2}}{2}}^{\infty} J(m) \sin 2 \pi m u \frac{d m}{m^{2}} \text {, }
$$

where the inner integral of the right side is

$$
\begin{aligned}
Q_{1}(u) & =\sum_{k=1}^{\infty} \frac{1}{\pi k} \int_{\frac{z}{2}}^{\infty} \frac{\sin 2 \pi k m \sin 2 \pi m u}{m^{2}} d m \\
& =\sum_{k=1}^{\infty} \frac{1}{2 \pi k} \int_{\frac{1}{2}}^{\infty} \frac{\cos 2 \pi(k-u) m-\cos 2 \pi(k+u) m}{m^{2}} d m \\
& =\frac{1}{2 \pi}\left(Q_{11}(u)-Q_{12}(u)\right) .
\end{aligned}
$$

We write

$$
\begin{aligned}
& Q_{11}=t \int_{1}^{\infty} \frac{\cos (t \log u)}{u^{\sigma+1}} Q_{11}(u) d u, \\
& Q_{12}=t \int_{1}^{\infty} \frac{\cos (t \log u)}{u^{\sigma+1}} Q_{12}(u) d u .
\end{aligned}
$$

3.1. Estimation of $Q_{12}$.

$$
\begin{aligned}
Q_{12}(u) & =\sum_{k=1}^{\infty} \frac{1}{k} \int_{\frac{1}{2}}^{\infty} \frac{\cos 2 \pi(k+u) m}{m^{2}} d m \\
& =\sum_{k=1}^{\infty}\left(1+\frac{u}{k}\right) \int_{(k+u) / 2}^{\infty} \frac{\cos 2 \pi m}{m^{2}} d m
\end{aligned}
$$

and then

$$
\begin{aligned}
Q_{12}=t \sum_{k=1}^{\infty} \int_{1}^{\infty} \frac{\cos (t \log u)}{u^{\sigma+1}} d u \int_{(k+u) / 2}^{\infty} \frac{\cos 2 \pi m}{m^{2}} d m \\
\quad+\sum_{k=1}^{\infty} \frac{t}{k} \int_{1}^{\infty} \frac{\cos (t \log u)}{u^{\sigma}} d u \int_{(k+u) / 2}^{\infty} \frac{\cos 2 \pi m}{m^{2}} d m \\
=Q_{121}+Q_{122},
\end{aligned}
$$

where 


$$
\begin{aligned}
Q_{121} & =\sum_{k=1}^{\infty} \int_{0}^{\infty} \frac{\cos v}{e^{\sigma v / t}} d v \int_{\left(k+e^{v / t}\right) / 2}^{\infty} \frac{\cos 2 \pi m}{m^{2}} d m \\
& =\sum_{k=1}^{t} \int_{(k+1) / 2}^{\infty} \frac{\cos 2 \pi m}{m^{2}} d m \int_{0}^{t \log (2 m-k)} \frac{\cos v}{e^{\sigma v / t}} d v \\
& =o\left(\sum_{k=1}^{t} \int_{(k+1) / 2}^{\infty} \frac{d m}{2}\right)+o\left(\sum_{k=m+1}^{\infty} \int_{0}^{\infty} \frac{\cos v}{e^{\sigma v / t}} d v \int_{\left(k+e^{v / t}\right) / 2}^{\infty} \frac{\cos 2 \pi m}{m^{2}} d m\right. \\
& \left.=O(\log t)+O(1)=O(\log t), \frac{d v}{k^{2}} \int_{0}^{\infty v / t}\right)
\end{aligned}
$$

and

$$
\begin{aligned}
Q_{122} & =\sum_{k=1}^{\infty} \frac{t}{k} \int_{(k+1) / 2}^{\infty} \frac{\cos 2 \pi m}{m^{2}} d m \int_{1}^{2 m-k} \frac{\cos (t \log u)}{u^{\sigma}} d u \\
& =\sum_{k=1}^{\infty} \frac{1}{k} \int_{(k+1) / 2}^{\infty} \frac{\cos 2 \pi m}{m^{2}} d m \int_{0}^{t \log (2 m-k)} e^{(1-\sigma) v / t} \cos v d v \\
& =o\left(\sum_{k=1}^{\infty} \frac{1}{k} \int_{(k+1) / 2}^{\infty} \frac{(2 m-k)^{1-\sigma}}{m^{2}} d m\right)=o(1) .
\end{aligned}
$$

Therefore $Q_{12}=O(\log t)$ as $t \rightarrow \infty$.

3.2. Estimation of $Q_{11}$.

$$
Q_{11}=t \int_{1}^{\infty} \frac{\cos (t \log u)}{u^{\sigma+1}}\left(\sum_{k=1}^{\infty} \frac{1}{k} \int_{\frac{1}{2}}^{\infty} \frac{\cos 2 \pi(k-u) m}{m^{2}} d n\right) d u \text {. }
$$

Since

$$
\int_{\frac{1}{2}}^{\infty} \frac{\cos 2 \pi(k-u) m}{m^{2}} d m=|k-u| \int_{|k-u| / 2}^{\infty} \frac{\cos 2 \pi m}{m^{2}} d m
$$

is bounded for $|k-u|<1$ and $O(1 /|k-u|)$ for $|k-u|>1$, we can interchange the order of surmation and integration on the right side; that is 


$$
\begin{aligned}
Q_{11} & =\sum_{k=1}^{\infty} \frac{t}{k}\left(\int_{1}^{k} \frac{\cos (t \log u)}{u^{\sigma+1}} d u \int_{\frac{1}{2}}^{\infty} \frac{\cos 2 \pi(k-u) m}{m^{2}} d m\right. \\
& \left.+\int_{k}^{\infty} \frac{\cos (t \log u)}{u^{\sigma+1}} d u \int_{\frac{2}{2}}^{\infty} \frac{\cos 2 \pi(u-k) m}{m^{2}} d m\right) \\
& =Q_{111}+Q_{112},
\end{aligned}
$$

where

$$
\begin{aligned}
& Q_{111}=\sum_{k=2}^{\infty} \frac{t}{k} \iint_{0}^{(k-1) / 2} \frac{\cos 2 \pi m}{m^{2}} d m \int_{k-2 m}^{k} \frac{(k-u) \cos (t \log u)}{u^{\sigma+1}} d u \\
& \left.+\int_{(k-1) / 2}^{\infty} \frac{\cos 2 \pi m}{m^{2}} d m \int_{1}^{k} \frac{(k-u) \cos (t \log u)}{u^{\sigma+1}} d u\right) \\
& =\sum_{k=2}^{\infty} \frac{1}{k} \int_{0}^{(k-1) / 2} \frac{\cos 2 \pi m}{m^{2}} d m \int_{t \log (k-2 m)}^{t \log k} \frac{k-e^{v / t}}{e^{\sigma v / t}} \cos v d v \\
& +0\left(\sum_{k=2}^{\infty}\left|\int_{(k-1) / 2}^{\infty} \frac{\cos 2 \pi m}{m^{2}} d m\right|\right) \\
& =0 \sum_{k=2}^{\infty} \frac{1}{k}\left\{\int_{0}^{k / 2 t} \frac{1}{m^{2}} \frac{m}{(k-2 m)} \frac{t m}{k} d m\right. \\
& \left.\left.+\left(\int_{k / 2}^{k / 4}+\int_{k / 4}^{(k-1) / 2}\right) \frac{1}{m^{2}} \frac{m}{(k-2 m)^{\sigma}} d m\right\}\right)+o(1) \\
& =0\left(\sum_{k=2}^{\infty} \frac{1}{k^{1+\sigma}}+\sum_{k=2}^{\infty} \frac{1}{k^{1+\sigma}} \int_{k / 2 t}^{k / 4} \frac{d m}{m}+\sum_{k=2}^{\infty} \frac{1}{k^{2}} \int_{k / 4}^{(k-1) / 2} \frac{d m}{(k-2 m)^{\sigma}}\right)+o(1) \\
& =O(\log t) \text {, }
\end{aligned}
$$

and

$$
\begin{aligned}
Q_{112} & =\sum_{k=1}^{\infty} \frac{t}{k} \int_{k}^{\infty} \frac{\cos (t \log u)}{u^{\sigma+1}} d u \int_{(u-k) / 2}^{\infty} \frac{(u-k) \cos 2 \pi m}{m^{2}} d m \\
& =\sum_{k=1}^{\infty} \frac{1}{k} \int_{0}^{\infty} \frac{\cos 2 \pi m}{m^{2}} d m \int_{t \log k}^{t \log (k+2 m)} \frac{\left(e^{v / t}-k\right) \cos v}{e^{\sigma v / t}} d v \\
& =0\left(\sum_{k=1}^{\infty} \frac{1}{k}\left\{\int_{0}^{k / 2 t} \frac{1}{m^{2}} \frac{t m}{k} \frac{m}{(m+2 k)^{\sigma}} d m+\iint_{k / 2 t}^{k}+\int_{k}^{\infty} \frac{1}{m^{2}} \frac{m}{(m+2 k)^{\sigma}} d m\right\}\right) \\
& =o(\log t) .
\end{aligned}
$$

Thus we have proved that $Q_{11}=O(\log t)$ and then 


$$
Q_{1}=Q_{11}-Q_{12}=O(\log t) \text {. }
$$

It remains to estimate $Q_{2}$.

$$
\begin{aligned}
& \text { 4. Estimation of } Q_{2} \\
& Q_{2}=t \int_{\frac{z_{2}}{2}}^{\infty} \frac{J(m)}{m} d m \int_{1}^{\infty} \cos (t \log u) \cos 2 \pi m u \frac{d u}{u^{\sigma}} \\
& =t \int_{1}^{\infty} \frac{\cos (t \log u)}{u^{\sigma}} d u \int_{\frac{1}{2}}^{\infty} \frac{J(m)}{m} \cos 2 \pi m u d m \text {, }
\end{aligned}
$$

which will be proved in the Appendix. The inner integral of the right side is

$$
\begin{aligned}
Q_{2}(u) & =\sum_{k=1}^{\infty} \frac{1}{\pi k} \int_{\frac{1}{2}}^{\infty} \sin 2 \pi k m \cos 2 \pi m u \frac{d m}{m} \\
& =\sum_{k=1}^{\infty} \frac{1}{2 \pi k} \int_{\frac{1}{2}}^{\infty}\{\sin 2 \pi(k+u) m+\sin 2 \pi(k-u) m\} \frac{d m}{m} \\
& =Q_{21}(u)+Q_{22}(u) .
\end{aligned}
$$

We write

$$
\begin{aligned}
Q_{2}= & t \int_{1}^{\infty} \frac{\cos (t \log u)}{u^{\sigma}}\left(Q_{21}(u)+Q_{22}(u)\right) d u \\
= & Q_{21}+Q_{22} . \\
& \text { 5. Estimation of } Q_{21}
\end{aligned}
$$

We get

$$
\begin{aligned}
Q_{21} & =\sum_{k=1}^{\infty} \frac{t}{2 \pi k} \int_{1}^{\infty} \frac{\cos (t \log u)}{u^{\sigma}} d u \int_{(k+u) / 2}^{\infty} \frac{\sin 2 \pi m}{m} d m \\
& =\sum_{k=1}^{\infty} \frac{1}{2 \pi k} \int_{(k+1) / 2}^{\infty} \frac{\sin 2 \pi m}{m} d m \int_{0}^{t \log (2 m-k)} e^{(1-\sigma) v / t} \cos v d v \\
& =\sum_{k=1}^{\infty} \frac{1}{4 \pi k} \int_{(k+1) / 2}^{\infty} \frac{d m}{m} \int_{0}^{t \log (2 m-k)}\{\sin (2 \pi m+v)+\sin (2 \pi m-v)\} e^{(1-\sigma) v / t} d v \\
& =\frac{1}{4 \pi}\left(Q_{211}+Q_{212}\right) .
\end{aligned}
$$


5.1. Estimation of $Q_{211}$. By the transformation $2 \pi m+v=w$, $Q_{211}=\sum_{k=1}^{\infty} \frac{1}{k} \int_{(k+1) / 2}^{\infty} \frac{d m}{m} \int_{2 \pi m}^{t \log (2 m-k)+2 \pi m} e^{(1-\sigma)(w-2 \pi m) / t} \sin w d \omega$.

The function $w=t \log (2 m-k)+2 \pi m$ is an increasing function of $m$ on the interval $((k+1) / 2, \infty)$ and then its inverse function $m=M(w)$ is also increasing on the interval $((k+1) \pi, \infty)$; therefore

$$
Q_{211}=\sum_{k=1}^{\infty} \frac{1}{k} \int_{(k+1)}^{\infty} \sin w d \omega\left(e^{(1-\sigma) w / t} \int_{M(\omega)}^{\omega / 2 \pi} \frac{d m}{m e^{2 \pi(1-\sigma) m / t}}\right) .
$$

If we denote by $y(w)$ the function in the bracket on the right side, that is,

$$
y(w)=e^{(1-\sigma) w / t} \int_{M(\omega)}^{\omega / 2 \pi} \frac{d m}{m e^{2 \pi(1-\sigma) m / t}},
$$

then $y(w)$ is non-negative on the interval $((k+1) \pi, \infty)$ and vanishes at both ends of the interval. For, by the second mean-value theorem,

$$
\begin{aligned}
y(w) & =\frac{e^{(1-\sigma) w / t}}{M(w)} \int_{M(\omega)}^{\theta} \frac{d m}{e^{2 \pi m(1-\sigma) / t}}(M(\omega)<\theta<w / 2 \pi) \\
& \leq \frac{t}{2 \pi(1-\sigma) M(\omega)} e^{(1-\sigma)(w-2 \pi M(\omega)) / t} \\
& =\frac{t(2 M(\omega)-k)^{1-\sigma}}{2 \pi(1-\sigma) M(w)}=o(1) \text { as } w \rightarrow \infty,
\end{aligned}
$$

since

$$
w=t \log (2 M(w)-k)+2 \pi M(w)
$$

Now

$$
\begin{aligned}
& y^{\prime}(w)=\frac{1-\sigma}{t} y(w)+\frac{1}{w}-\frac{M^{\prime}(w) e^{(1-\sigma) \omega / t}}{M(\omega) e^{2 \pi(1-\sigma) M(w) / t}} \\
& =\frac{1-\sigma}{t} y(w)+\frac{1}{w}-\frac{M^{\prime}(w)(2 M(w)-k)^{1-\sigma}}{M(w)} \\
& =\frac{(2 M(\omega)-k)^{1-\sigma}}{2 \pi M(\omega)}-\frac{M^{\prime}(w)(2 M(\omega)-k)^{1-\sigma}}{M(\omega)}-\frac{1}{2 \pi} e^{(1-\sigma) \omega / t} \int_{M(\omega)}^{w / 2 \pi} \frac{d m}{m^{2} e^{2 \pi m(1-\sigma) / t}} \\
& =\frac{t M^{\prime}(\omega)}{\pi M(\omega)(2 M(\omega)-k)}-\frac{1}{2 \pi} e^{(1-\sigma) \omega / t} \int_{M(\omega)}^{\omega / 2 \pi} \frac{d m}{m^{2} e^{2 \pi(1-\sigma) m / t}},
\end{aligned}
$$


using (1) at the first step, integration by parts at the second step, and using the relation

$$
M^{\prime}(w)=1 /\left(2 \pi+\frac{2 t}{2 M(w)-k}\right)
$$

at the last step. Therefore

$$
\begin{aligned}
Q_{211} & =\sum_{k=1}^{\infty} \frac{1}{k} \int_{(k+1) \pi}^{\infty} y^{\prime}(w) \cos w d \omega \\
& =\sum_{k=1}^{\infty} \frac{t}{k \pi} \int_{(k+1) \pi}^{\infty} \frac{M^{\prime}(w) \cos w}{M(\omega)(2 M(\omega)-k)^{\sigma}} d \omega \\
& \quad-\sum_{k=1}^{\infty} \frac{1}{2 \pi k} \int_{(k+1) \pi}^{\infty} e^{(1-\sigma) \omega / t} \cos d \omega \int_{M(\omega)}^{\omega / 2 \pi} \frac{d m}{m^{2} e^{2 \pi(1-\sigma) m / t}} \\
& =\frac{1}{\pi} R_{1}-\frac{1}{2 \pi} R_{2} .
\end{aligned}
$$

We shall first estimate $R_{1}$. By (2),

$$
\begin{aligned}
M(w)(2 M(w)-k)^{\sigma} / M^{\prime}(w) & =M(w)(2 M(w)-k)^{\sigma}(2 \pi+2 t /(2 M(w)-k)) \\
& =M(w)(2 \pi(2 M(w)-k)+2 t) /(2 M(w)-k)^{1-\sigma} .
\end{aligned}
$$

Consider the function of $x$ :

$$
z(x)=x(2 \pi(2 x-k)+2 t) /(2 x-k)^{1-\sigma} ;
$$

then its logarithmic differential coefficient is

$$
\begin{aligned}
\frac{z^{\prime}(x)}{z(x)} & =\frac{1}{x}+\frac{4 \pi}{2 \pi(2 x-k)+2 t}-\frac{2(1-\sigma)}{2 x-k} \\
& =z_{0}(x) / x(2 \pi(2 x-k)+2 t)(2 x-k),
\end{aligned}
$$

where

$$
\begin{aligned}
z_{0}(x) & =(2 x-k)(2 \pi(2 x-k)+2 t)+4 \pi x(2 x-k)-2(1-\sigma) x(2 \pi(2 x-k)+2 t) \\
& =8 \pi(1+\sigma) x^{2}-4((2+\sigma) \pi k-\sigma t) x+2 k(\pi k-t),
\end{aligned}
$$

which vanishes at

$$
\begin{aligned}
x_{0} & =\frac{1}{4 \pi(1+\sigma)}\left\{(2+\sigma) \pi k-\sigma t+\sqrt{((2+\sigma) \pi k-\sigma t)^{2}-4 \pi(1+\sigma) k(\pi k-t)}\right\} \\
& \leq k .
\end{aligned}
$$

Therefore, $z(x)$ takes a minimum between $(k+1) / 2$ and $k$ or is monotone 
increasing in the interval of $x:((k+1) / 2, \infty)$. For each case

$$
R_{I}=0\left(\sum_{k=1}^{\infty} \frac{1}{k^{1+\sigma}}\right)=O(1) \text {. }
$$

The integral in the $k$ th term of $R_{2}$ is

$$
\begin{aligned}
R_{2}(k) & =\int_{(k+1) \pi}^{\infty} e^{(1-\sigma) \omega / t} \cos \omega d \omega \int_{M(\omega)}^{\omega / 2} \frac{d m}{m^{2} e^{2 \pi(1-\sigma) m / t}} \\
& =\int_{(k+1) / 2}^{\infty} \frac{d m}{m^{2} e^{2 \pi(1-\sigma) m / t}} \int_{2 \pi m}^{2 \pi m+t \log (2 m-k)} e^{(1-\sigma) \omega / t} \cos \omega d \omega \\
& =0\left(\int_{(k+1) / 2}^{\infty} \frac{(2 m-k)^{1-\sigma}}{m^{2}} d m\right)=o\left(\frac{1}{k}\right)
\end{aligned}
$$

and then

$$
R_{2}=\sum_{k=1}^{\infty} \frac{1}{k} R_{2}(k)=0\left(\sum_{k=1}^{\infty} \frac{1}{k^{1+\sigma}}\right)=O(1) .
$$

Therefore,

$$
Q_{211}=O\left(\left|R_{1}\right|+\left|R_{2}\right|\right)=O(1)
$$

\subsection{Estimation of $Q_{212}$.}

$$
\begin{aligned}
Q_{212} & =\sum_{k=1}^{\infty} \frac{1}{k} \int_{(k+1) / 2}^{\infty} \frac{d m}{m} \int_{0}^{t \log (2 m-k)} e^{(1-\sigma) v / t} \sin (2 \pi m-v) d v \\
& =\sum_{k=1}^{\infty} \frac{1}{k} \int_{0}^{\infty} e^{(1-\sigma) v / t} d v \int_{\left(e^{v / t}+k\right) 2}^{\frac{\sin (2 \pi m-v)}{m}} d m \\
& =\sum_{k=1}^{\infty} \frac{1}{\pi k} \int_{0}^{\infty} e^{(1-\sigma) v / t} \frac{\cos \left(\left(e^{v / t}+k\right) \pi-v\right)}{e^{v / t}+k} \frac{1}{\sum^{2}} \frac{1}{2 \pi k} \int_{0}^{\infty} e^{(1-\sigma) v / t} d v \int_{k=1}^{\infty} d m \\
& =\frac{1}{\pi} S_{1}-\frac{1}{2 \pi} S_{2},
\end{aligned}
$$

where 


$$
\begin{aligned}
S_{2} & =\sum_{k=1}^{\infty} \frac{1}{k} \int_{(k+1) / 2}^{\infty} \frac{d m}{m^{2}} \int_{0}^{t \log (2 m-k)} e^{(1-\sigma) v / t} \cos (2 \pi m-v) d v \\
& =0\left(\sum_{k=1}^{\infty} \frac{1}{k} \int_{(k+1) / 2}^{\infty} \frac{(2 m-k)^{1-\sigma}}{m^{2}} d m\right)=O(1) .
\end{aligned}
$$

Now

$$
\begin{aligned}
S_{1} & =\sum_{k=1}^{\infty} \frac{(-1)^{k}}{k} \int_{0}^{\infty} \frac{e^{(1-\sigma) v / t}}{e^{v / t}+k} \cos \left(\pi e^{v / t}-v\right) d v \\
& =\sum_{k=1}^{\infty} \frac{1}{2 k} \int_{0}^{\infty} \frac{e^{(1-\sigma) v / t} \cos \left(\pi e^{v / t}-v\right)}{\left(e^{v / t}+2 k\right)\left(e^{v / t}+2 k+1\right)} d v \\
& +\sum_{k=1}^{\infty} \frac{1}{2 k(2 k+1)} \int_{0}^{\infty} \frac{e^{(1-\sigma) v / t} \cos \left(\pi e^{v / t}-v\right)}{e^{v / t}+2 k+1} d v-\int_{0}^{\infty} \frac{e^{(1-\sigma) v / t}}{e^{v / t}+1} \cos \left(\pi e^{v / t}-v\right) d v \\
& =S_{11}+S_{12}-S_{13} .
\end{aligned}
$$

We consider the function $x(v)=\pi e^{v / t}-v$, which has two roots $v_{1}$ and $v_{3}$ such that $\pi<v_{1}=\pi+O(I / t)$ and $v_{3}$ satisfies the relations

$$
v_{3}=t \log \left(v_{3} / \pi\right), v_{3}>t \log (t / \pi)
$$

and

$$
\begin{aligned}
t \log \frac{t}{\pi} & <t \log \left(\frac{t}{\pi} \log \frac{t}{\pi}\right)<t \log \left(\frac{t}{\pi} \log \left(\frac{t}{\pi} \log \frac{t}{\pi}\right)\right)<\ldots \\
& <v_{3}=t\left\{\log \frac{t}{\pi}+\log \log \frac{t}{\pi}\left(1+\frac{1+o(1)}{\log (t / \pi)}\right)\right\} .
\end{aligned}
$$

We write

$$
\begin{aligned}
S_{11} & =\sum_{k=1}^{\infty} \frac{1}{2 k}\left(\int_{0}^{v_{1}}+\int_{v_{1}}^{v_{2}}+\int_{v_{2}}^{v_{3}}+\int_{v_{3}}^{\infty}\right) d v \\
& =s_{111}+s_{112}+s_{113}+s_{114},
\end{aligned}
$$

where $v_{2}=\frac{4}{5} t \log \frac{t}{\pi}$ and

$$
S_{111}=0\left(\sum_{k=1}^{\infty} \frac{1}{k^{3}}\right)=O(1) .
$$


We shall use the transformation $w=v-\pi e^{v / t}$ for the estimation of $s_{112}$. We denote it by $w=w(v)$ and its inverse function by $v=v(w)$. Then

$$
S_{112}=\sum_{k=1}^{\infty} \frac{1}{2 k} \int_{w\left(v_{1}\right)}^{w\left(v_{2}\right)} \frac{e^{(1-\sigma) v(w) / t} \cos w d \omega}{\left(e^{v(w) / t}+2 k\right)\left(e^{v(w) / t}+2 k+1\right)\left(1-\pi e^{v(w) / t} / t\right)} .
$$

For large $t$ and for $w\left(v_{1}\right)<w<w\left(v_{2}\right)$,

$$
1-\frac{\pi}{t} e^{v(w) / t}>1-(\pi / t) e^{v_{2} / t}=1-\frac{\pi}{t}\left(\frac{t}{\pi}\right)^{4 / 5}>\frac{1}{2},
$$

and the function of $x: y(x)=x^{1-\sigma} /(x+2 k)(x+2 k+1) \quad(x>0)$ takes its maximum at the point

$$
x_{0}=\frac{1}{2(1+\sigma)}\left\{\sqrt{(4 k+1)^{2}-\left(1-\sigma^{2}\right)}+\sigma(4 k+1)\right\} \cong 2 k+\frac{1}{2} .
$$

Therefore,

$$
\begin{aligned}
S_{112} & =0\left(\sum_{k=1}^{(t / \pi)^{4 / 5}} \frac{y\left(x_{0}\right)}{k}\right)+o\left(\sum_{k=(t / \pi)^{4 / 5}+1}^{\infty} \frac{y\left(e^{v^{2 / t}}\right)}{k}\right) \\
& =o\left(\sum_{k=1}^{(t / \pi)^{4 / 5}} \frac{1}{k} \cdot \frac{1}{k^{1+\sigma}}\right)+o\left(\sum_{k=(t / \pi)^{4 / 5}}^{\infty} \frac{t^{(1-\sigma) 4 / 5}}{k^{3}}\right) \\
& =O(1),
\end{aligned}
$$

and

$$
\begin{aligned}
S_{113} & =o\left(\sum_{k=1}^{\infty} \frac{1}{k} \frac{t^{(1-\sigma) 4 / 5}}{\left(t^{4 / 5}+k\right)^{2}} v_{3}\right) \\
& =o\left(\frac{\log t}{t^{(4 \sigma-1) / 5}} \sum_{k=1}^{4 / 5} \frac{1}{k}\right)+o\left(t^{(9-4 \sigma) / 5} \log t \sum_{k=t^{4 / 5}+1}^{\infty} \frac{1}{k^{3}}\right) \\
& =o(1) .
\end{aligned}
$$

Using the transformation

$$
w=\pi e^{v / t}-v, \quad d v=\left((\pi / t) e^{v / t}-1\right) d v
$$

for the integral of $S_{114}$, we get 


$$
S_{114}=o\left(\sum_{k=1}^{\infty} \frac{1}{k} \frac{e^{(1-\sigma) v_{3} / t}}{\left(e^{v_{3} / t}+k\right)^{2}} \frac{1}{\log (t / \pi)}\right)=O(1)
$$

Thus we have proved that $S_{11}=O(1)$. We shall now estimate

$$
\begin{aligned}
S_{12}= & \sum_{k=1}^{\infty} \frac{1}{2 k(2 k+1)} \int_{0}^{\infty} \frac{e^{(1-\sigma) v / t} \cos \left(\pi e^{v / t}-v\right)}{e^{v / t}+2 k+1} d v \\
= & \sum_{k=1}^{\infty} \frac{1}{2 k(2 k+1)} \iint_{0}^{v 1}+\int_{v_{1}}^{v 2}+\int_{v_{2}}^{t \log (t / \pi)-\sqrt{t}} \\
& +\int_{t \log (t / \pi)-\sqrt{t}}^{t \log (t / \pi)}+\int_{t \log (t / \pi)}^{t \log (t / \pi)+\sqrt{t}}+\int_{t \log (t / \pi)+\sqrt{t}}^{v_{3}}+\int_{v_{3}}^{\infty} d v \\
= & \sum_{i=1}^{7} S_{12 i} .
\end{aligned}
$$

We have

$$
S_{121}=0\left(\sum_{k=1}^{\infty} \frac{1}{k^{2}} \frac{1}{k}\right)=O(1)
$$

Since the function $y(x)=x^{1-\sigma} /(x+2 k+1)$ takes its maximum at the point $x=(1-\sigma) /(2 k+1) / \sigma$,

$$
S_{122}=0\left(\sum_{k=1}^{L} \frac{1}{k^{2+\sigma}}\right)+0\left(\sum_{k=L+1}^{\infty} \frac{t^{(1-\sigma) 4 / 5}}{k^{3}}\right)=0(1),
$$

where $L=\frac{\sigma}{2(1-\sigma)}\left(\frac{t}{\pi}\right)^{4 / 5}-\frac{1}{2}$, using the transformation $w=v-\pi e^{v / t}$

For the estimation of $S_{123}$, we use the transformation

$$
\begin{gathered}
w=w(v)=v-\pi e^{v / t}, \\
d v=\left(1-(\pi / t) e^{v / t}\right) d v .
\end{gathered}
$$

By $v=v(w)$ we denote the inverse function of $w=w(v)$ in the interval $\left(v_{2}, t \log (t / \pi)-\sqrt{t}\right)$. Then the integral of the $k$ th term of $s_{123}$

becomes 


$$
\begin{aligned}
S_{123} & =\int_{v_{2}}^{v_{2}^{\prime}} \frac{e^{(1-\sigma) v / t} \cos \left(v-\pi e^{v / t}\right)}{e^{v / t}+2 k+1} d v \\
& =\int_{w\left(v_{2}\right)}^{w\left(v_{2}^{\prime}\right)} \frac{e^{(1-\sigma) v(w) / t} \cos w}{\left(e^{v(w) / t}+2 k+1\right)\left(1-(\pi / t) e^{v(w) / t}\right)} d w,
\end{aligned}
$$

where $v_{2}^{\prime}=t \log (t / \pi)-\sqrt{t}$. We consider the function of $x:$

$$
y=y(x)=x^{1-\sigma} /(x+2 k+1)(1-\pi x / t),
$$

whose logarithmic differential coefficient is

$$
\begin{aligned}
\frac{y^{\prime}}{y} & =\frac{1-\sigma}{x}-\frac{1}{x+2 k+1}+\frac{1}{(t / \pi)-x} \\
& =\frac{(1+\sigma) x^{2}+\sigma(2 k+1-t / \pi) x+(1-\sigma)(2 k+1) t / \pi}{x(x+2 k+1)((t / \pi)-x)} .
\end{aligned}
$$

If $2 k+1>t / \pi$, then $y^{\prime}>0$. In the case $t / \pi \leq 2 k+1$, the discriminant of the number on the right side is

$$
\begin{aligned}
\sigma^{2}(2 k+1-t / \pi)^{2}-4\left(1-\sigma^{2}\right) & (2 k+1) t / \pi \\
= & \sigma^{2}\left(\left(\frac{t}{\pi}-\frac{1-\sqrt{1-\sigma^{2}}}{\sigma}\right)^{2}(2 k+1)\right)\left(\frac{t}{\pi}-\left(\frac{1+\sqrt{1-\sigma^{2}}}{\sigma}\right)^{2}(2 k+1)\right),
\end{aligned}
$$

which is negative for

$$
\frac{t}{\pi} \geq 2 k+1>\left(\frac{1+\sqrt{1-\sigma^{2}}}{\sigma}\right)^{-2} \frac{t}{\pi}=\left(\frac{1-\sqrt{1-\sigma^{2}}}{\sigma}\right)^{2} \frac{t}{\pi} .
$$

Therefore, if

$$
k>\frac{1}{2}\left(\frac{1-\sqrt{1-\sigma^{2}}}{\sigma}\right)^{2} \frac{t}{\pi}-\frac{1}{2}=k_{0},
$$

then the function $y\left(e^{v(w) / t}\right)$ increases in the interval $\left(w\left(v_{2}\right), w\left(v_{2}^{\prime}\right)\right)$ and 


$$
\begin{aligned}
S_{123}(k) & =o\left(\frac{e^{(1-\sigma) v_{2}^{\prime} / t}}{\left(e^{v_{2}^{\prime} / t}+2 k+1\right)\left(1-(\pi / t) e^{v_{2}^{\prime} / t}\right)}\right) \\
& =o\left(\frac{t^{1-\sigma} e^{-(1-\sigma) / \sqrt{t}}}{\left(t e^{-1 / \sqrt{t}}+2 k+1\right)\left(1-e^{-1 / \sqrt{t}}\right)}\right) \\
& =o\left(\frac{t^{1-\sigma} \sqrt{t}}{t}\right)=O(1),
\end{aligned}
$$

and then

$$
\sum_{k=k_{0}+1}^{\infty} \frac{1}{2 k(2 k+1)} S_{123}(k)=0(1) .
$$

In the case $k<k_{0}$, the numerator of the right side of (3) has two roots $x^{\prime}$ and $x^{\prime \prime} \quad\left(x^{\prime}<x^{\prime \prime}\right)$ and

$$
\begin{aligned}
x^{\prime \prime} & =\frac{1}{2(1+\sigma)}\left\{\sigma\left(\frac{t}{\pi}-(2 k+1)\right)+\sqrt{\sigma^{2}\left(\frac{t}{\pi}-(2 k+1)\right)^{2}-4\left(1-\sigma^{2}\right)(2 k+1) \frac{t}{\pi}}\right\} \\
& \leq \frac{\sigma}{1+\sigma}\left(\frac{t}{\pi}-(2 k+1)\right) .
\end{aligned}
$$

Writing $x^{\prime \prime}=e^{v^{\prime \prime} / t}$,

$$
\begin{aligned}
v^{\prime \prime} & \leq t \log \left(\frac{t}{\pi}-(2 k+1)\right)-t \log \frac{1+\sigma}{\sigma} \leq v_{2}^{\prime}, \\
x^{\prime} & =\frac{1}{2(1+\sigma)}\left\{\sigma\left(\frac{t}{\pi}-(2 k+1)\right)-\sqrt{\sigma^{2}\left(\frac{t}{\pi}-(2 k+1)\right)^{2}-4\left(1-\sigma^{2}\right)(2 k+1) \frac{t}{\pi}}\right\} \\
& \cong \frac{(1-\sigma)(2 k+1) t / \pi}{\sigma(t / \pi-(2 k+1))} .
\end{aligned}
$$

If $k>(2 \sigma-1) / 2(1-\sigma)$, then $x^{\prime}>1$, and then, writing $x^{\prime}=e^{v^{\prime} / t}$,

$$
\begin{aligned}
S_{123}(k) & =o\left(\frac{e^{(1-\sigma) v^{\prime} / t}}{\left(e^{v^{\prime} / t}+2 k+1\right)\left(1-(\pi / t) e^{v^{\prime} / t}\right)}\right)+o\left(\frac{e^{(1-\sigma) v_{2}^{\prime} / t}}{\left(e^{v_{2}^{\prime} / t}+2 k+1\right)\left(1-(\pi / t) e^{v_{2}^{\prime} / t}\right.}\right) \\
& =O(1),
\end{aligned}
$$

and then

$$
\sum_{k=(2 \sigma-1) / 2(1-\sigma)+1}^{k_{0}} \frac{1}{2 k(2 k+1)} S_{123}(k)=O(1) .
$$


If $I \leq k \leq(2 \sigma-1) / 2(1-\sigma)$, then $v^{\prime} \leq 0$. Similarly as above $S_{123}(k)=O(1)$ for such $k$. Thus we have proved that $S_{123}$ is bounded.

The function $y(v)=e^{(1-\sigma) v / t} /\left(e^{v / t}+2 k+1\right)$ takes a maximum at $v=t \log ((1-\sigma)(2 k+1) / \sigma)$ and its maximum value is $O\left(1 / k^{\sigma}\right)$. Writing

$$
k_{2}=\frac{\sigma t}{2(1-\sigma) \pi}-\frac{1}{2}
$$

and

$$
k_{1}=\frac{t}{2(1-\sigma) \pi e^{1 / \sqrt{t}}}-\frac{z}{2} \cong k_{2}-\frac{\sigma \sqrt{t}}{2(1-\sigma) \pi} \text {, }
$$

we get

$$
\begin{aligned}
S_{124} & =o\left(\sum_{k=1}^{\infty} \frac{1}{k^{2}} \sqrt{t} \max _{v_{2}^{\prime} \leq v \leq t \log (t / \pi)} y(v)\right) \\
& =0\left(\sum_{k=1}^{k} \frac{1}{k^{2}} \frac{\sqrt{t} t^{1-\sigma}}{t}+\sum_{k=k_{1}+1}^{k_{2}} \frac{1}{k^{2}} \frac{\sqrt{t}}{k^{\sigma}}+\sum_{k=k_{2}+1}^{\infty} \frac{1}{k^{2}} \frac{\sqrt{t} t^{1-\sigma}}{t}\right) \\
& =O(1) .
\end{aligned}
$$

$S_{125}$ and $S_{126}$ become bounded by similar estimations. Putting $w=w(v)=\pi e^{v / t}-v$ and denoting by $v=v(w)$ the solution of the equation in the interval $\left(v_{3}, \infty\right)$, the integral of the $k$ th term of $S_{127}$ is

$$
\begin{aligned}
\int_{v_{3}}^{\infty} \frac{e^{(1-\sigma) v / t}}{e^{v / t}+2 k+1} \cos \left(\pi e^{v / t}-v\right) d v & =\pi \int_{0}^{\infty} \frac{(\omega+v(\omega))^{1-\sigma} \cos \omega d \omega}{(\omega+v(\omega)+(2 k+1) \pi)\left((\pi / t) e^{v(w) / t}-1\right)} \\
& =o\left(\int_{0}^{2 \pi} \frac{d \omega}{(\omega+v(\omega))^{\sigma} \log (t / \pi)}\right)=o(1),
\end{aligned}
$$

which shows that $S_{127}$ is also bounded.

Summing up the above estimate, we see that $S_{12}$ is bounded. Since $S_{13}$ is also bounded by an estimation similar to $S_{12}, S_{1}$ is bounded. Combining with the estimation of $S_{2}$, we get 


$$
Q_{212}=O(1)
$$

Combining the estimation of $\$ 5.1$ and $\$ 5.2$, we get

$$
Q_{21}=O(1) \text {. }
$$

Therefore it remains to estimate $Q_{22}$.

\section{Estimation of $Q_{22}$}

$$
\begin{aligned}
& Q_{22}=\sum_{k=1}^{\infty} \frac{t}{2 \pi k}\left\{\int_{1}^{k} \frac{\cos (t \log u)}{u^{\sigma}} d u \int_{(k-u) / 2}^{\infty} \frac{\sin 2 \pi m}{m} d m\right. \\
& \left.-\int_{k}^{\infty} \frac{\cos (t \log u)}{u^{\sigma}} d u \int_{(u-k) / 2}^{\infty} \frac{\sin 2 \pi m}{m} d m\right\} \\
& =\sum_{k=2}^{\infty} \frac{t}{2 \pi k}\left\{\int_{0}^{k-1} \frac{\cos (t \log (k-u))}{(k-u)^{\sigma}} d u \int_{u / 2}^{\infty} \frac{\sin 2 \pi m}{m} d m\right. \\
& \left.-\int_{0}^{\infty} \frac{\cos (t \log (k+u))}{(k+u)^{\sigma}} d u \int_{u / 2}^{\infty} \frac{\sin 2 \pi m}{m} d m\right\} \\
& -\frac{t}{2 \pi} \int_{1}^{\infty} \frac{\cos (t \log u)}{u^{\sigma}} d u \int_{(u-1) / 2}^{\infty} \frac{\sin 2 \pi m}{m} d m \\
& =\sum_{k=2}^{\infty} \frac{t}{2 \pi k} \int_{0}^{K}\left\{\frac{\cos (\log (k-u))}{(k-u)^{\sigma}}-\frac{\cos (t \log (k+u))}{(k+u)^{\sigma}}\right\} d u \int_{u / 2}^{\infty} \frac{\sin 2 \pi m}{m} d m \\
& +\sum_{k=2}^{\infty} \frac{t}{2 \pi k} \int_{K}^{k-1} \frac{\cos (\log (k-u))}{(k-u)^{\sigma}} d u \int_{u / 2}^{\infty} \frac{\sin 2 \pi m}{m} d m \\
& -\sum_{k=2}^{\infty} \frac{t}{2 \pi k} \int_{K}^{\infty} \frac{\cos (t \log (k+u))}{(k+u)^{\sigma}} d u \int_{u / 2}^{\infty} \frac{\sin 2 \pi m}{m} d n \\
& -\frac{t}{2 \pi} \int_{1}^{\infty} \frac{\cos (t \log u)}{u^{\sigma}} d u \int_{(u-1) / 2}^{\infty} \frac{\sin 2 \pi m}{m} d m \\
& =\frac{1}{2 \pi}\left(Q_{221}+Q_{222}{ }^{-Q_{223}}{ }^{-Q_{224}}\right) \text {, }
\end{aligned}
$$

where $K(<k-1)$ will be determined later.

By the transformations $t \log (k-u)=v$ and $t \log (k+u)=v$, we get 


$$
\begin{aligned}
& Q_{221}= \\
& =\sum_{k=2}^{\infty} \frac{t}{k} \int_{0}^{K}\left\{\frac{\cos (t \log (k-u))}{(k-u)^{\sigma}}-\frac{\cos (t \log (k+u))}{(k+u)^{\sigma}}\right\} d u \int_{u / 2}^{\infty} \frac{\sin 2 \pi m}{m} d n \\
& =\sum_{k=2}^{\infty} \frac{1}{k}\left\{\int_{t \log (k-u)}^{t \log k} e^{(1-\sigma) v / t} \cos v d v \int_{\left(k-e^{v / t}\right) / 2}^{\infty} \frac{\sin 2 \pi m}{m} d m\right. \\
& \left.-\int_{t \log k}^{t \log (k+K)} e^{(1-\sigma) v / t} \cos v d v \int_{\left(e^{v / t}-k\right) / 2}^{\infty} \frac{\sin 2 \pi m}{m} d m\right\} \\
& =\sum_{k=2}^{\infty} \frac{1}{k}\left\{\int_{0}^{-t \log (1-K / k)} e^{-(1-\sigma) w / t} \cos (t \log k-\omega) d \omega \int_{k\left(1-e^{-\omega / t}\right) / 2}^{\infty} \frac{\sin 2 \pi m}{m} d m\right. \\
& \left.-\int_{0}^{t \log (1+K / k)} e^{(1-\sigma) \omega / t} \cos (t \log k+\omega) d \omega \int_{k\left(e^{\omega / t}-1\right) / 2}^{\infty} \frac{\sin 2 \pi m}{m} d m\right\} \\
& =\sum_{k=2}^{\infty} \frac{1}{k^{\sigma}}\left\{\int _ { 0 } ^ { t \operatorname { l o g } ( 1 + K / k ) } \left[e^{-(1-\sigma) w / t} \cos (t \log k-w) \int_{k\left(1-e^{-w / t}\right) / 2}^{\infty} \frac{\sin 2 \pi m}{m} d m\right.\right. \\
& \left.-e^{(1-\sigma) w / t} \cos (t \log k+w) \int_{k\left(e^{w / t}-1\right) / 2}^{\infty} \frac{\sin 2 \pi m}{m} d m\right) d \omega \\
& \left.+\int_{t \log (1+K / k)}^{-t \log (1-K / k)} e^{-(1-\sigma) w / t} \cos (t \log k-w) d w \int_{k\left(1-e^{-w / t}\right) / 2}^{\infty} \frac{\sin 2 \pi m}{m} d m\right\} \\
& =\sum_{k=2}^{\infty} \frac{1}{k^{\sigma}}\left\{\int_{0}^{t \log (1+K / k)}(\cos (t \log k-\omega)-\cos (t \log k+\omega)) e^{-(1-\sigma) \omega / t} d \omega\right. \\
& \cdot \int_{k\left(1-e^{-w / t}\right) / 2}^{\infty} \frac{\sin 2 \pi m}{m} d m+\int_{0}^{t \log (1+K / k)} \cos (t \log k+w) \\
& \text { - }\left(e^{-(1-\sigma) \omega / t} \int_{k\left(1-e^{-w / t}\right) / 2}^{\infty} \frac{\sin 2 \pi m}{m} d m-e^{(1-\sigma) \omega / t} \int_{k\left(e^{w / t}-1\right) / 2}^{\infty} \frac{\sin 2 \pi m}{m} d m\right) d \omega \\
& \left.+\int_{t \log (1+K / k)}^{-t \log (1-K / k)} \cos (t \log k-\omega) e^{-(1-\sigma) \omega / t} d \omega \int_{k\left(1-e^{-\omega / t}\right) / 2}^{\infty} \frac{\sin 2 \pi m}{m} d m\right\} \\
& =2 T_{1}+T_{2}+T_{3} \text {. }
\end{aligned}
$$




\section{Estimation of $T_{1}$}

$$
\begin{aligned}
& T_{1}=\sum_{k=2}^{\infty} \frac{\sin (\log k)}{k^{\sigma}} \int_{0}^{t \log (1+K / k)} e^{-(1-\sigma) \omega / t} \sin \omega \omega \omega \int_{k\left(1-e^{-\omega / t}\right) / 2}^{\infty} \frac{\sin 2 \pi m}{m} d m \\
& =\sum_{k=2}^{\infty} \frac{\sin (t \log k)}{k^{\sigma}} \\
& \text { - }\left\{\int_{0}^{K / 2(1+K / k)} \frac{\sin 2 \pi m}{m} d m \int_{0}^{-t \log (1-2 m / k)} e^{-(1-\sigma) w / t} \sin w d \omega\right. \\
& \left.+\int_{K / 2(1+K / k)}^{\infty} \frac{\sin 2 \pi m}{m} d m \int_{0}^{t \log (1+K / k)} e^{-(1-\sigma) \omega / t} \sin w d v\right\} \\
& =T_{11}+T_{12} \text {, } \\
& T_{11}=\int_{3 / 2}^{\infty} \frac{\sin (t \log k)}{k^{\sigma}}(d k+d J(k)) \\
& \text { - } \int_{0}^{K / 2(1+K / k)} \frac{\sin 2 \pi m}{m} d n \int_{0}^{-t \log (1-2 m / k)} e^{-(1-\sigma) \omega / t} \sin w d w \\
& =T_{111}+T_{112} \text {. } \\
& \text { 7.1. Estimation of } T_{111} \text {. We take } \\
& K=k^{\sigma-\varepsilon} \quad(0<\varepsilon<\sigma) ;
\end{aligned}
$$

then

$$
\begin{aligned}
T_{111} & =\int_{0}^{c_{0}} \frac{\sin 2 \pi m}{m} d m \int_{3 / 2}^{\infty} \frac{\sin (t \log k)}{k^{\sigma}} d k \int_{0}^{-t \log (1-2 m / k)} e^{-(1-\sigma) w / t} \sin w d w \\
& +\int_{c_{0}}^{\infty} \frac{\sin 2 \pi m}{m} d m \int_{M(m)}^{\infty} \frac{\sin (t \log k)}{k^{\sigma}} d k \int_{0}^{-t \log (1-2 m / k)} e^{-(1-\sigma) w / t} \sin w d \omega \\
& =U_{1}+U_{2}
\end{aligned}
$$

where $c_{0}=(3 / 2)^{\sigma-\varepsilon} / 2\left(1+(3 / 2)^{\sigma-\varepsilon-1}\right)$ and $M=M(m)$ is the solution of the equation $m=k^{\sigma-\varepsilon} / 2\left(1+k^{\sigma-\varepsilon-1}\right)$; then $M \cong(2 m)^{1 /(\sigma-\varepsilon)}$. We shall first estimate $U_{1}$. By the formula 


$$
\left(1+a^{2}\right) \int_{0}^{b} e^{a w} \sin w a \omega=1-e^{a b} \cos b+a e^{a b} \sin b
$$

with $a=-(1-\sigma) / t$ and $b=-t \log (1-2 m / k)$, the inner double integral of $U_{1}$ becomes

$$
\begin{aligned}
\int_{3 / 2}^{\infty} & \frac{\sin (t \log k)}{k^{\sigma}} d k \int_{0}^{-t \log (1-2 m / k)} e^{-(1-\sigma) \omega / t} \sin w d \omega \\
& =\left(1+\frac{(1-\sigma)^{2}}{t^{2}}\right)^{-1} \int_{3 / 2}^{\infty} \frac{\sin (t \log k)}{k^{\sigma}}\left\{\left(1-\left(1-\frac{2 m}{k}\right)^{1-\sigma} \cos \left(t \log \left(1-\frac{2 m}{k}\right)\right)\right\}\right. \\
& =\left(1+\frac{(1-\sigma)^{2}}{t^{2}}\right)^{-1}\left(U_{11}-(1-\sigma) U_{12}\right) .
\end{aligned}
$$

Now

$$
\begin{aligned}
& U_{11}=\int_{3 / 2}^{\infty} \frac{\sin (t \log k)}{k^{\sigma}}(1-\cos (t \log (1-2 m / k))) d k+o(1) \\
& =\int_{3 / 2}^{t^{2 /(1+\sigma)}} \frac{\sin (t \log k)}{k^{\sigma}}(1-\cos (t \log (1-2 m / k))) d k+o(1) \\
& =\frac{1}{2} \int_{3 / 2}^{t^{2 /(1+\sigma)}}\{2 \sin (t \log k)-\sin (t \log (k-2 m)\} \\
& \left.-\sin \left(t \log \left(t \log \left(k^{2} /(k-2 m)\right)\right)\right)\right\} \frac{d k}{k^{\sigma}}+o(1) \\
& =U_{111}-U_{112}-U_{113} \text {. }
\end{aligned}
$$

where

$$
\begin{aligned}
U_{111} & =\frac{1}{t} \int_{t \log (3 / 2)}^{(2 /(1+\sigma)) t \log t} e^{(1-\sigma) k / t} \sin k d k \\
& =o\left[\frac{1}{t} t^{2(1-\sigma) /(1+\sigma)}\right]=o(1)
\end{aligned}
$$

and similarly $U_{112}$ and $U_{113}$ are bounded. Therefore $U_{11}=O(1)$. Since $U_{12}$ is also bounded, we have proved that $U_{1}$ is bounded. 
Now, the inner double integral of $U_{2}$ is $U_{2}(m)=$

$$
\begin{aligned}
& =\int_{M(m)}^{\infty} \frac{\sin (t \log k)}{k^{\sigma}} d k \int_{0}^{-t \log (1-2 m / k)} e^{-(1-\sigma) w / t} \sin w d \omega \\
& =\left(1+\frac{(1-\sigma)^{2}}{t^{2}}\right)^{-1} \int_{M(m)}^{\infty} \frac{\sin (t \log k)}{k^{\sigma}}\left\{\left(1-\left(1-\frac{2 m}{k}\right)^{1-\sigma} \cos \left(t \log \left(1-\frac{2 m}{k}\right)\right)\right\}\right. \\
& =\left(1+\frac{(1-\sigma)^{2}}{t^{2}}\right)^{-1}\left(U_{21}(m)-(1-\sigma) U_{22}(m)\right),
\end{aligned}
$$

where

$$
\begin{aligned}
U_{21}(m) & =\int_{M(m)}^{\infty} \frac{\sin (t \log k)}{k^{\sigma}}\left\{1-\cos \left(t \log \left(1-\frac{2 m}{k}\right)\right)\right\} d k+o\left(\frac{1}{\varepsilon /(\sigma-\varepsilon)}\right) \\
& =0\left(\frac{t^{2} m^{2}}{m(1+\sigma) /(\sigma-\varepsilon)}\right)+o\left(\frac{1}{m^{\varepsilon /(\sigma-\varepsilon)}}\right)
\end{aligned}
$$

and

$$
U_{22}(m)=o\left(\frac{m}{m^{\sigma /(\sigma-\varepsilon)}}\right)=o\left(\frac{I}{\varepsilon /(\sigma-\varepsilon)}\right) \text {. }
$$

Therefore, writing $a=2(\sigma-\varepsilon) /(1-\sigma+2 \varepsilon)$,

$$
\begin{aligned}
U_{2} & =\left(1+\frac{(1-\sigma)^{2}}{t^{2}}\right)^{-1} \int_{c_{0}}^{t^{a}} \frac{\sin 2 \pi m}{m} d m \\
& =\frac{1}{2}\left(1+\frac{(1-\sigma)^{2}}{t^{2}}\right)^{-1} \int_{c_{0}}^{t^{a}} \frac{\sin 2 \pi m}{m} d m \int_{M(m)}^{(m t)^{1 / \sigma}}\left\{\frac{\sin (t \sin (t \log k)-\sin (t \log (k-2 m))}{k^{\sigma}}\left\{1-\cos \left(t \log \left(1-\frac{2 m}{k}\right)\right]\right\} d k+o(1)\right. \\
& =\frac{1}{2}\left(1+\frac{(1-\sigma)^{2}}{t^{2}}\right)^{-1}\left(U_{21}+U_{22}\right)+o(\log t) ;
\end{aligned}
$$




$$
\begin{aligned}
U_{21}=\frac{1}{t} \int_{c_{0}}^{t^{a}} \frac{\sin 2 \pi m}{m} d m\left\{\int_{t \log M(m)}^{(1 / \sigma) t \log (m t)}\right. & e^{(1-\sigma) k / t} \sin k d k \\
& \left.-\int_{t \log (M(m)-2 m)}^{t \log \left((m t)^{1 / \sigma}-2 m\right)} \frac{e^{k / t}}{\left(e^{k / t}+2 m\right)^{\sigma}} \sin k d k\right\} \\
=\frac{1}{t} \int_{c_{0}}^{t^{a}} \frac{\sin 2 \pi m}{m} d m\left\{\int_{t \log M(m)}^{(1 / \sigma) t \log (m t)}\right. & \left.+\frac{1}{e^{\sigma k / t}}-\frac{1}{\left(e^{i / t}+2 m\right)}\right) e^{i / t} \sin k d k \\
& +\int_{t \log \left((m t)^{1 / \sigma}-2 m\right)}^{(1 / \sigma) t \log (m t)} \frac{e^{k / t}}{\left(e^{k / t}+2 m\right)^{\sigma}} \sin k d k \\
& \left.-\int_{t \log (M(m)-2 m)}^{t \log M(m)} \frac{e^{k / t}}{\left(e^{k / t}+2 m\right)^{\sigma}} \sin k d k\right\} \\
U_{211}+U_{212}-U_{213} . &
\end{aligned}
$$

Since

$$
x^{1-\sigma}-\frac{x}{(x+m)^{\sigma}}=-\sum_{j=1}^{\infty}\left(\begin{array}{c}
-\sigma \\
j
\end{array}\right) \frac{m^{j}}{x^{j-1+\sigma}},
$$

we get, writing $x=e^{k / t}$,

$$
\begin{aligned}
U_{211} & =o\left(\frac{1}{t} \int_{c_{0}}^{t^{a}}\left(\sum_{j=1}^{\infty}\left|\left(\begin{array}{c}
-\sigma \\
j
\end{array}\right)\right| \frac{m^{j}}{M(m)^{j-1+\sigma}}\right) \frac{d n}{m}\right) \\
& =o\left(\frac{1}{t} \int_{c_{0}}^{t^{a}} \frac{d m}{m^{\sigma /(\sigma-\varepsilon)}}+\frac{1}{t} \sum_{j=2}^{\infty}\left|\left(\begin{array}{c}
-\sigma \\
j
\end{array}\right)\right| \int_{c_{0}}^{t^{a}} \frac{d m}{m^{(j-1)(1-\sigma+\varepsilon) /(\sigma-\varepsilon)+\sigma /(\sigma-\varepsilon)}}\right) \\
& =O(1) .
\end{aligned}
$$

Since the function $y=x /(x+2 m)^{\sigma}$ is increasing,

$$
U_{212}=o\left(\frac{1}{t} \int_{c_{0}}^{t^{a}} \frac{1}{m}(m t)^{(1-\sigma) / \sigma} \frac{1}{(m t)^{(1-\sigma) / \sigma}} d m\right)=O(1)
$$

and

$$
U_{213}=0\left(\frac{1}{t} \int_{c_{0}}^{t^{a}} \frac{1}{m} M(m)^{1-\sigma} \frac{d n}{M(m)} d m\right)=0\left(\int_{c_{0}}^{t^{a}} \frac{d m}{m^{\sigma /(\sigma-\varepsilon)}}\right)=O(1)
$$


Thus we have proved that $U_{21}$ is bounded. Similarly $U_{22}$ is bounded, and then

$$
U_{2}=o(\log t)
$$

Therefore, $T_{111}$ is also of order $O(\log t)$.

7.2. Estimation of $T_{112}$.

$$
\begin{aligned}
T_{112}=\int_{3 / 2}^{\infty} \frac{\sin (t \log k)}{k^{\sigma}} d J(k) & \\
& \cdot \int_{0}^{K / 2(1+K / k)} \frac{\sin 2 \pi m}{m} d m \int_{0}^{-t \log (1-2 m / k)} e^{-(1-\sigma) w / t} \sin w d \omega .
\end{aligned}
$$

We use the formula: if $f$ is absolutely continuous and $f^{\prime}$ is of bounded variation in the range of integration, then

$$
\begin{aligned}
\int f(k) d J(k) & =-\int f^{\prime}(k) J(k) d k \\
& =-\sum_{j=1}^{\infty} \frac{1}{\pi j} \int f^{\prime}(k) \sin 2 \pi j k d k=2 \sum_{j=1}^{\infty} \int f(k) \cos 2 \pi j k d k,
\end{aligned}
$$

where both limits of integration are half of odd integers. Then

$$
\begin{aligned}
T_{112} & =2 \sum_{j=1}^{\infty} \int_{3 / 2}^{\infty} \frac{\sin (t \log k) \cos 2 \pi j k}{k^{\sigma}} d k \\
& \cdot \int_{0}^{K / 2(1+K / k)} \frac{\sin 2 \pi m}{m} d m \int_{0}^{-t \log (1-2 m / k)} e^{-(1-\sigma) \omega / t} \sin w d w \\
& =2\left(1-\frac{(1-\sigma)^{2}}{t^{2}}\right)_{j=1}^{-1} \int_{3 / 2}^{\infty} \frac{\sin (t \log k) \cos 2 \pi j k}{k^{\sigma}} d k \\
\cdot \int_{0}^{K / 2(1+K / k)} \frac{\sin 2 \pi m}{m}\left\{\left(1-\left(1-\frac{2 m}{k}\right)^{1-\sigma} \cos \left(t \log \left(1-\frac{2 m}{k}\right)\right)\right)\right. & \left.-\frac{1-\sigma}{t}\left(1-\frac{2 m}{k}\right)^{1-\sigma} \sin \left(t \log \left(1-\frac{2 m}{k}\right)\right)\right\} d m \\
& =2\left(1-\frac{(1-\sigma)^{2}}{t^{2}}\right)^{-1}\left(V_{1}-(1-\sigma) V_{2}\right) ;
\end{aligned}
$$




$$
\begin{aligned}
& V_{1}=\sum_{j=1}^{\infty} \int_{0}^{c_{0}} \frac{\sin 2 \pi m}{m} d m \\
& \text { - } \int_{3 / 2}^{\infty} \frac{\sin (t \log k) \cos 2 \pi j k}{k^{\sigma}}\left(1-\left(1-\frac{2 m}{k}\right)^{1-\sigma} \cos \left(t \log \left(1-\frac{2 m}{k}\right)\right)\right) d k \\
& +\sum_{j=1}^{\infty} \int_{c_{0}}^{\infty} d m \int_{M(m)}^{\infty} d k \\
& =V_{11}+V_{12} \text {. } \\
& \text { 7.2.1. Estimation of } V_{11} \text {. } \\
& V_{11}=\frac{3}{4} \sum_{j=1}^{\infty} \int_{0}^{c_{0}} \frac{\sin 2 \pi m}{m} d m \\
& \text { - } \int_{3 / 2}^{\infty}\left\{2 \sin (t \log k+2 \pi j k)-\left(1-\frac{2 m}{k}\right)^{1-\sigma} \sin (t \log (k-2 m)+2 \pi j k)\right. \\
& -\left(1-\frac{2 m}{\bar{k}}\right)^{1-\sigma} \sin \left(t \log \left(k^{2} /(k-2 m)\right)+2 \pi j k\right) \\
& +2 \sin (t \log k-2 \pi j k)-\left(1-\frac{2 m}{k}\right)^{1-\sigma} \sin (t \log (k-2 m)-2 \pi j k) \\
& -\left(1-\frac{2 m}{k}\right)^{1-\sigma} \sin \left(t \log \left(k^{2} /(k-2 m)\right)-2 \pi j k\right) \frac{d k}{k^{\sigma}} \\
& =\frac{3}{4} \int_{0}^{c_{0}} \frac{\sin 2 \pi m}{m}\left(2 V_{111}-V_{112}-V_{113}+2 V_{114}-V_{115}-V_{116}\right) d m \text {. }
\end{aligned}
$$

We use the transformation $p=t \log k+2 \pi j k$ and denote by $k(p)$ the solution of $k$ of this equation for fixed $p$. Then

$$
\begin{aligned}
V_{111} & =\sum_{j=1}^{\infty} \int_{3 \pi j+t \log 3 / 2}^{\infty} \frac{\sin p d p}{k(p)^{\sigma}(2 \pi j+t / k(p))} \\
& =\sum_{j=1}^{\infty} \frac{(-1)^{j+1} \cos (t \log 3 / 2)}{(3 / 2)^{\sigma}(2 \pi j+2 t / 3)}+\sum_{j=1}^{\infty} \int_{3 \pi j+t \log 3 / 2}^{\infty} \frac{(1-\sigma) k(p) \cos p}{k(p)^{\sigma}(2 \pi j k(p)+t)} d p \\
& +\sum_{j=1}^{\infty} \int_{3 \pi j+t \log 3 / 2}^{\infty} \frac{2 \pi j k(p) k(p)^{1-\sigma} \cos p}{(2 \pi j k(p)+t)^{2}} d p \\
= & O(1),
\end{aligned}
$$


since $k^{\prime}(p)=1 / \frac{d p}{d k}=1 /(2 \pi j+t / k(p))$. Similarly $V_{112}$ and $V_{113}$ are also bounded.

For the estimation of $V_{114}$, we use the transformation

$$
p=|t \log k-2 \pi j k| \text {. }
$$

The curves

$$
y=t \log k \text { and } y=2 \pi j k
$$

touch each other at the point $k=e$ for $j=t / 2 \pi e$. The lines $y=2 \pi j k(j>t / 2 \pi e)$ do not intersect the curve $y=t \log k$, but the lines $y=2 \pi j k \quad(j<t / 2 \pi e)$ intersect the curve $y=t \log k$. If $j<\frac{t}{3 \pi} \log 3 / 2$, then they intersect at only one point $k_{j}$ in the range $(3 / 2, \infty)$ such that

$$
\frac{k_{j}}{\log k_{j}}=\frac{t}{2 \pi j}
$$

that is,

$$
k_{j}=\frac{t}{2 \pi j} \log \left(\frac{t}{2 \pi j} \log \frac{t}{2 \pi j}\right)+o\left(\frac{\log \log (t / 2 \pi j)}{(\log (t / 2 \pi j))^{2}}\right) .
$$

Further, the curve (4) takes its maximum at the point $k=t / 2 \pi j$. If $\frac{t}{3 \pi} \log 3 / 2 \leq j<\frac{t}{2 \pi e}$, then the curves (5) intersect at two points in the range $(3 / 2, \infty)$, one being less than $e$ and the other greater than $e$. If $t / 2 \pi e \leq j<t / 3 \pi$, then the curve (4) becomes

$$
p=2 \pi j k-t \log k
$$

and takes a minimum between $3 / 2$ and $e$. If $j \geq t / 3 \pi$, then the curve (6) increases; that is, $2 \pi j-t / k>0$. We write

$$
\begin{aligned}
& V_{114}=\left(\sum_{j=1}^{(t / 3 \pi) \log (3 / 2)}+\sum_{j=(t / 3 \pi) \log (3 / 2)+1}^{t / 3 \pi}+\sum_{j=t / 3 \pi+1}^{\infty}\right) \\
& \text { - } \int_{3 / 2}^{\infty} \frac{\sin (t \log k-2 \pi, j k)}{k^{\sigma}} d k \\
& =W_{1}+w_{2}+w_{3} \text {. }
\end{aligned}
$$


Using the transformation, (6) and denoting by $k(p)$ the solution of the equation (6) for fixed $p$,

$$
\begin{aligned}
W_{3} & =-\sum_{j=t / 3 \pi+1}^{\infty} \int_{3 / 2}^{\infty} \frac{\sin (2 \pi j k-t \log k)}{k^{\sigma}} d k \\
& =-\sum_{j=t / 3 \pi+1}^{\infty} \int_{3 \pi j-t \log 3 / 2}^{\infty} \frac{\operatorname{sinpdp}}{k(p)^{\sigma}(2 \pi j-t / k(p))} \\
& =\sum_{j=t / 3 \pi+1}^{\infty} \frac{(-1)^{j} \cos (t \log 3 / 2)}{(3 / 2)^{\sigma}(2 \pi j-2 t / 3)}-\sum_{j=t / 3 \pi+1}^{\infty} \int_{3 \pi j-t \log 3 / 2}^{\infty} \frac{(1-\sigma) k^{\prime}(p) \cos p}{k(p)^{\sigma}(2 \pi j k(p)-t)} d p \\
& =o(1), \sum_{j=t / 3 \pi+1}^{\infty} \int_{3 \pi j-t \log 3 / 2}^{\infty} \frac{2 \pi j k^{\prime}(p) k(p)^{1-\sigma} \cos p}{(2 \pi j k(p)-t)^{2}} d p
\end{aligned}
$$

since $k^{\prime}(p)=I /(2 \pi j-t / k(p))$.

$$
\begin{aligned}
w_{1} & =\sum_{j=1}^{(t / 3 \pi) \log (3 / 2)}\left(\int_{3 / 2}^{k}+\int_{k_{j}}^{\infty}\right) \frac{\sin (t \log k-2 \pi j k)}{k^{\sigma}} d k \\
& =w_{11}+w_{12} .
\end{aligned}
$$

By the transformation (6),

$$
\begin{aligned}
& W_{12}=\sum_{j=1}^{(t / 3 \pi) \log (3 / 2)} \int_{2 \pi j k_{j}-t \log k_{j}}^{\infty} \frac{\sin p d p}{k(p)^{\sigma}(2 \pi j-t / k(p))} \\
& =0\left(\sum_{j=1}^{(t / 3 \pi) \log (3 / 2)} \frac{1}{(t / j)} \sigma_{j}\right)=o(1) \text {. }
\end{aligned}
$$

Now we shall estimate

$$
\begin{aligned}
W_{2} & =\sum_{j=(t / 3 \pi)}^{t / 3 \pi} \sum_{\log (3 / 2)+1}^{\infty} \frac{\sin (t \log k-2 \pi j k)}{k^{\sigma}} d k \\
& =\sum_{j=(t / 3 \pi) \log (3 / 2)+1}^{t / 3 \pi}\left(\int_{3 / 2}^{10}+\int_{10}^{\infty}\right) d k \\
& =W_{21}+W_{22} .
\end{aligned}
$$

For $(t / 3 \pi)<j<(t / 3 \pi) \log (3 / 2)$, we have

$$
(3 / 2) / \log (3 / 2)<(t / 2 \pi j)<(3 / 2)
$$


and then $k_{j}<10$. By the transformation $p=2 \pi j k-t \log k$,

$$
\begin{aligned}
w_{22} & =\sum_{j=(t / 3 \pi) \log (3 / 2)+1}^{t / 3 \pi} \int_{20 j \pi-t \log 10}^{\infty} \frac{\text { sinpdp }}{k(p)^{\sigma}(2 \pi j-t / k(p))} \\
& =0\left[\begin{array}{cc}
t / 3 \pi & \frac{1}{j} \\
j=(t / 3 \pi) \log (3 / 2)+1 & =o(1)
\end{array}\right.
\end{aligned}
$$

and

$$
\begin{aligned}
W_{21} & =\int_{[(t / 3 \pi) \log (3 / 2)]+\frac{1}{2}}^{[t / 3 \pi]+\frac{1}{2}}(d j+d J(j)) \int_{3 / 2}^{10} \frac{\sin (t \log k-2, j k)}{k^{\sigma}} d k \\
& =W_{211}+W_{212}
\end{aligned}
$$

where

$$
\begin{aligned}
W_{211} & =\int_{[(t / 3 \pi) \log (3 / 2)]+\frac{\pi}{2}}^{[t / 3 \pi]+\frac{\pi}{2}} d j \int_{3 / 2}^{10} \frac{\sin (t \log k) \cos 2 \pi j k-\cos (t \log k) \sin 2 \pi j k}{k^{\sigma}} d k \\
& =\int_{3 / 2}^{10} \frac{\sin (t \log k)}{k^{\sigma}} d k \int_{[(t / 3 \pi) \log (3 / 2)]+\frac{1}{2}}^{[t / 3 \pi]+\frac{1}{2}} \cos 2 \pi j k d j \\
& -\int_{3 / 2}^{10} \frac{\cos (t \log k)}{k^{\sigma}} d k \int_{[(t / 3 \pi) \log (3 / 2)]+\frac{1}{2}}^{[t / 3 \pi]+\frac{1}{2}} \sin 2 \pi j k d j \\
& =O(1),
\end{aligned}
$$

and similarly $W_{212}=O(\log t)$. Thus we have proved that $W_{2}=O(\log t)$.

Now we shall estimate $V_{115}$ :

$$
\begin{aligned}
v_{115} & =\sum_{j=1}^{\infty} \int_{3 / 2}^{\infty}\left(1-\frac{2 m}{k}\right)^{1-\sigma} \frac{\sin (t \log (k-2 m)-2 \pi j k)}{k^{\sigma}} d k \\
& \left.=\sum_{j=1}^{t / 3 \pi}+\sum_{j=t / 3 \pi+1}^{\infty}\right) \int_{3 / 2}^{\infty} d k \\
& =W_{4}+w_{5}, \\
w_{5}= & t /\left(3-4 c_{0}\right) \pi \\
j=t / 3 \pi+1 & \left.\left(\int_{3 / 2}^{3 / 2+2 m}+\int_{3 / 2+2 m}^{\infty}\right) d k+\sum_{j=t /\left(3-4 c_{0}\right) \pi+1}^{\infty}\right]_{3 / 2}^{\infty} d k \\
= & w_{51}+w_{52}+W_{53} .
\end{aligned}
$$

For $W_{51}$, change the order of summation and integration and use the order 
of the integral of Dirichlet and conjugate Dirichlet kernels; then we can easily see that it is $O(\log t)$. For $W_{52}$ and $W_{53}$, we use the transformation $p=2 \pi j k-t \log (k-2 m)$, then $d p / d k>0$ and the method used for $V_{11 l}$ can be applied to them. Thus we get $W_{5}=O(\log t)$. Now

$$
\begin{aligned}
W_{4} & =\sum_{j=1}^{t / 3 \pi} \int_{3 / 2}^{\infty}\left(1-\frac{2 m}{k}\right)^{1-\sigma} \frac{\sin (t \log (k-2 m)-2 \pi j k)}{k^{\sigma}} d k \\
& =\sum_{j=1}^{t / 3 \pi}\left(\int_{3 / 2}^{t / 2 \pi j+2 m}+\int_{t / 2 \pi j+2 m}^{k_{j}^{\prime}}+\int_{k_{j}^{\prime}}^{\infty}\right) d k \\
& =W_{41}+W_{42}+W_{43} .
\end{aligned}
$$

where $k_{j}^{\prime}$ is the solution of the equation $k=\frac{t}{2 \pi j} \log (k-2 m)$, greater than $t / 2 \pi_{j}+2 m$; that is,

$$
\begin{aligned}
& k_{j}^{\prime}=\frac{t}{2 \pi j} \log \left(\frac{t}{2 \pi j} \log \frac{t}{2 \pi j}\right)-\frac{2 m}{\log (t / 2 \pi j)}+o\left(\frac{1}{(\log (t / 2 \pi j))^{2}}\right) ; \\
& W_{4 I}=\sum_{j=1}^{t / 3 \pi} \int_{3 / 2-2 m}^{t / 2 \pi j}\left(1-\frac{2 m}{k+2 m}\right)^{1-\sigma} \frac{\sin (t \log k-2 \pi j(k+2 m))}{(k+2 m)^{\sigma}} d k ;
\end{aligned}
$$

and then

$$
\begin{aligned}
\int_{0}^{c_{0}} \frac{\sin 2 \pi m}{m} W_{41} d m & \sum_{j=1}^{t / 3 \pi}\left\{\int_{0}^{c_{0}} \frac{\sin 2 \pi m}{m} \cos 4 \pi j m d m \int_{3 / 2-m}^{t / 2 \pi j}\left(1-\frac{2 m}{k+2 m}\right)^{1-\sigma} \frac{\sin (t \log k-2 \pi j k)}{(k+2 m)^{\sigma}} d k\right. \\
& \left.-\int_{0}^{c_{0}} \frac{\sin 2 \pi m}{m} \sin 4 \pi j m d m \int_{3 / 2-2 m}^{t / 2 \pi j}\left(1-\frac{2 m}{k+2 m}\right)^{1-\sigma} \frac{\cos (t \log k-2 \pi j k)}{(k+2 m)^{\sigma}} d k\right\} \\
= & W_{411}-W_{4 I 2}, \sum_{j=1}^{t / 3 \pi}\left\{\left[\frac{\sin 2 \pi m}{m} \frac{\sin 4 \pi j m}{4 \pi j} \int_{3 / 2-2 m}^{t / 2 \pi j}\left(1-\frac{2 m}{k+2 m}\right)^{1-\sigma} \frac{\sin (t \log k-2 \pi j k)}{(k+2 m)^{\sigma}} d k\right]_{m=0}^{c} 0\right. \\
W_{411} & -\int_{0}^{c_{0}}\left[\frac{\sin 2 \pi m}{m}\right)^{\prime} \frac{\sin 4 \pi j m}{4 \pi j} d m \int_{3 / 2-2 m}^{t / 2 \pi j}\left(1-\frac{2 m}{k+2 m}\right)^{1-\sigma} \frac{\sin (t \log k-2 \pi j k)}{(k+2 m)^{\sigma}} d k
\end{aligned}
$$




$$
\begin{aligned}
& -2(-1)^{j} \int_{0}^{c_{0}} \frac{\sin 2 \pi m}{m} \frac{\sin 4 \pi j m}{4 \pi j}(1-4 m / 3)^{1-\sigma} \frac{\sin (t \log (3 / 2-2 m)+4 \pi j m)}{(3 / 2)^{\sigma}} d m \\
& \left.+2 \int_{0}^{c_{0}} \frac{\sin 2 \pi m}{m} \frac{\sin 4 \pi j m}{4 \pi j} d m \int_{3 / 2-2 m}^{t / 2 \pi j} \frac{k^{1-\sigma}}{(k+2 m)^{2}} \sin (t \log k-2 \pi j k) d k\right\} .
\end{aligned}
$$

Now we put $p=t 208 k-2 \pi j k$; then $p$ increases as $k$ increases in the interval $(3 / 2-2 m, t / 2 \pi j)$, and the function

$$
y(k)=k^{2-\sigma} /(k+2 m)(t / k-2 \pi j)
$$

increases as $k$ increases. If $k=t / 2 \pi j$, then $p=t \log (t / 2 \pi j e)$. Suppose $k=t / 2 \pi j-\theta(\theta>0)$; then

$$
\begin{aligned}
p & =t \log (t / 2 \pi j-\theta)-t+2 \pi j \theta \\
& =t \log (t / 2 \pi j e)+t \log (1-2 \pi j \theta / t)+2 \pi j \theta .
\end{aligned}
$$

If we take $\theta=\frac{1}{j} \sqrt{t / \pi}$, we see that $p$ increases more than $2 \pi$ when $k$ increases from $t / 2 \pi j-\sqrt{t / \pi} / j$ to $t / 2 \pi j$. Therefore

$$
\begin{aligned}
\int_{3 / 2-2 m}^{t / 2 \pi j} \frac{k^{1-\sigma}}{k+2 m} \sin (t \log k-2 \pi j k) d k & =o\left(\frac{1}{j} \sqrt{t / \pi} \frac{1}{(t / 2 \pi j)^{\sigma}}\right) \\
& =O\left(1 / t^{\sigma-\frac{1}{2}} j^{1-\sigma}\right),
\end{aligned}
$$

and then $W_{411}=O(\log t)$. Similarly $W_{412}=O(\log t)$. Therefore $W_{41}$ is of order $\log t$. Similarly $W_{42}$ is also of order $\log t$. Since $W_{43}$ is bounded, $W_{4}$ is of order $\log t$. Thus we have proved that

$$
\int_{0}^{c_{0}} \frac{\sin 2 \pi m}{m} V_{115}=O(\log t) .
$$

A similar estimate holds for $V_{116}$. Collecting the above estimations, we get

$$
\begin{aligned}
V_{11} & =\frac{2}{\pi} \int_{0}^{c_{0}} \frac{\sin 2 \pi m}{m} w_{11} d m+o(\log t) \\
& =\frac{2}{\pi} \sum_{j=1}^{(t / 3 \pi) \log (3 / 2)} \int_{0}^{c_{0}} \frac{\sin 2 \pi m}{m} d \pi \int_{3 / 2}^{k} \frac{\sin (t \log k-2 \pi j k)}{k^{\sigma}} d k+o(\log t) .
\end{aligned}
$$


190

Masako Izumi and Shin-ichi Izumi

7.2.2. Estimation of $V_{12}$. We shall estimate

$$
\begin{aligned}
& V_{12}=\sum_{j=1}^{\infty} \int_{c_{0}}^{\infty} \frac{\sin 2 \pi m}{m} d m \\
& \text { - } \int_{M(m)}^{\infty} \frac{\sin (t \log k) \cos 2 \pi j k}{k^{\sigma}}\left\{1-\left(1-\frac{2 m}{k}\right)^{1-\sigma} \cos \left(t \log \left(1-\frac{2 m}{k}\right)\right)\right\} d k \\
& =\frac{3}{4} \sum_{j=1}^{\infty} \int_{c_{0}}^{\infty} \frac{\sin 2 \pi m}{m} d m \\
& \text { - } \int_{M(m)}^{\infty}\left\{2 \sin (t \log k+2 \pi j k)-\left(1-\frac{2 m}{k}\right)^{1-\sigma} \sin (t \log (k-2 m)+2 \pi j k)\right. \\
& -\left(1-\frac{2 m}{k}\right)^{1-\sigma} \sin \left(t \log \left(k^{2} /(k-2 m)\right)+2 \pi j k\right) \\
& +2 \sin (t \log k-2 \pi j k)-\left(1-\frac{2 m}{k}\right)^{1-\sigma} \sin (t \log (k-2 m)-2 \pi j k) \\
& \left.-\left\{1-\frac{2 m}{k}\right)^{1-\sigma} \sin \left(t \log \left(k^{2} /(k-2 m)\right)-2 \pi j k\right)\right\} \frac{d k}{k^{\sigma}} \\
& =\frac{3}{4}\left(2 V_{121}-V_{122}-V_{123}+2 V_{124}-V_{125^{-}} V_{126}\right) \text {. }
\end{aligned}
$$

By the transformation $p=t \log k+2 j k$, used for $V_{111}$, we get

$$
\begin{aligned}
& V_{121}=\sum_{j=1}^{\infty} \int_{c_{0}}^{\infty} \frac{\sin 2 \pi m}{m} d m \int_{M(m)}^{\infty} \frac{\sin (t \log k+2 \pi j k)}{k^{\sigma}} d k \\
& =\sum_{j=1}^{\infty} \int_{c_{0}}^{\infty} \frac{\sin 2 \pi m}{m} d m \int_{t \log M(m)+2 \pi j M(m)}^{\infty} \frac{\sin p d p}{k(p)^{\sigma}(2 \pi j+t / k(p))} \\
& =\sum_{j=1}^{\infty} \int_{c_{0}}^{\infty} \frac{\sin 2 \pi m \cos (t \log M(m)+2 \pi j M(m))}{m M(m)^{\sigma}(2 \pi j+t / M(m))} d m
\end{aligned}
$$

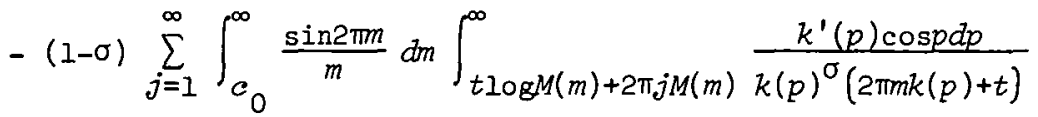

$$
\begin{aligned}
& -\sum_{j=1}^{\infty} 2 \pi j \int_{c_{0}}^{\infty} \frac{\sin 2 \pi m}{m} d m \int_{t \log M(m)+2 \pi j M(m)}^{\infty} \frac{k(p)^{l-\sigma_{k}}(p) \cos p}{(2 \pi j k(p)+t)^{2}} d p \\
& =x_{11}-x_{12}-x_{13} \text {, }
\end{aligned}
$$

https://doi.org/10.1017/S0004972700022577 Published online by Cambridge University Press 


$$
\begin{aligned}
& X_{I 1}=\frac{1}{2} \sum_{j=1}^{\infty} \int_{c_{0}}^{\infty}\{\sin (t \log M(m)+2 \pi j M(m)+2 \pi m) \\
& -\sin (t \log M(m)+2 \pi j M(m)-2 \pi m)\} \frac{d m}{m M(m)^{\sigma}(2 \pi j+t / M(m))} \\
& =\frac{2}{2}\left(x_{111}-X_{112}\right) \text {. }
\end{aligned}
$$

By the transformation $q=t \log M(m)+2 \pi j M(m)+2 \pi m$,

$$
\begin{aligned}
d q & =\left(t M^{\prime}(M) / M(m)+2 \pi j M^{\prime}(m)+2 \pi\right) d m \\
& \cong\left(\frac{t}{(\sigma-\varepsilon) m}+\frac{2^{1 /(\sigma-\varepsilon)} 2 \pi j m(1-\sigma+\varepsilon) /(\sigma-\varepsilon)}{\sigma-\varepsilon}+2 \pi\right) d m,
\end{aligned}
$$

since

$$
\begin{gathered}
M^{\prime}(m)-1 / \frac{d}{d k}\left(\frac{k^{\sigma-\varepsilon}}{2\left(1+k^{\sigma-\varepsilon-1}\right)}\right) \cong \frac{2 k^{1-\sigma+\varepsilon}}{\sigma-\varepsilon} \cong \frac{2(2 m)^{(1-\sigma+\varepsilon) /(\sigma-\varepsilon)}}{\sigma-\varepsilon}, \\
M^{\prime}(m) / M(m) \cong 1 / m(\sigma-\varepsilon),
\end{gathered}
$$

and then

$$
\begin{aligned}
X_{111} & =\sum_{j=1}^{\infty} \int_{t \log M\left(c_{0}\right)+2 \pi j M\left(c_{0}\right)+2 \pi c_{0}}^{\infty} \frac{\frac{1}{m(q) M(m(q))^{\sigma}(2 \pi j+t / M(m(q)))}}{\frac{1}{t M^{\prime}(m(q)) / M(m(q))+2 \pi j M^{\prime}(m(q))+2 \pi} \sin q d q} \\
& =o\left(\sum_{j=1}^{\infty} \frac{1}{j^{2}}\right)=O(1),
\end{aligned}
$$

since $m / M(m)^{1-\sigma}$ increases. We can see that $X_{112}$ is also bounded by a similar estimate, and then $X_{11}$ is bounded. For the estimation of $X_{12}$, use the relation $k^{\prime}(p)=1 /(2 \pi j+t / k(p))$ and that the function of $x$ : $y=x^{\perp-\sigma} /(2 \pi j x+t)^{2}$ takes its maximum at the point $x=(1-\sigma) t / 2(1+\sigma) \pi j$; then we can see that $x_{12}$ is bounded. $x_{13}$ is also similarly bounded. Therefore $V_{121}$ is bounded. Similarly $V_{122}$ and $V_{123}$ are bounded. We shall estimate $V_{124}$. 


$$
\begin{aligned}
V_{124} & =\sum_{j=1}^{\infty} \int_{c_{0}}^{\infty} \frac{\sin 2 \pi m}{m} d m \int_{M(m)}^{\infty} \frac{\sin (t \log k-2 \pi j k)}{k^{\sigma}} d k \\
& =\sum_{j=1}^{(t / 3 \pi) \log (3 / 2)}+\sum_{j=(t / 3 \pi) \log (3 / 2)}^{t / 3 \pi}+\sum_{j=t / 3 \pi+1}^{\infty} \\
& =x_{41}+x_{42}+x_{43} .
\end{aligned}
$$

By the transformation $p=2 \pi j k-t \log k$ and integration by parts, we get

$$
\begin{aligned}
& x_{43}=-\sum_{j=t / 3 \pi+1}^{\infty} \int_{c_{0}}^{\infty} \frac{\sin 2 \pi m}{m} d m \int_{2 \pi j M(m)-t \log M(m)}^{\infty} \frac{\sin p d p}{k(p)^{\sigma}(2 \pi j-t / k(p))} \\
& =\sum_{j=t / 3 \pi+1}^{\infty}\left\{\int_{c_{0}}^{\infty} \frac{\sin 2 \pi m}{m} \frac{\cos (2 \pi j M(m)-t \log M(m))}{M(m)^{\sigma}(2 \pi j-t / M(m))}\right. \\
& -\int_{c_{0}}^{\infty} \frac{\sin 2 \pi m}{m} d n \int_{2 \pi j M(m)-t \log M(m)}^{\infty} \frac{(1-\sigma) k^{\prime}(p) \cos p}{k(p)^{\sigma}(2 \pi j k(p)-t)} d p \\
& -\int_{c_{0}}^{\infty} \frac{\sin 2 \pi m}{m} d m \int_{2 \pi j M(m)-t \log M(m)}^{\infty} \frac{2 \pi j k(p)^{1-\sigma_{k^{\prime}}(p) \cos p}}{(2 \pi j k(p)-t)^{2}} d p \\
& =x_{431}-x_{432}-x_{433}, \\
& X_{431}=\sum_{j=t / 3 \pi+1}^{\infty} \int_{c_{0}}^{\infty} \frac{d m}{2 m M(m)^{\sigma}(2 \pi j-t / M(m))} \\
& \{\sin (2 \pi j M(m)-t \log M(m)+2 \pi m)-\sin (2 \pi j M(m)-t \log M(m)-2 \pi m)\} \\
& =x_{4311}-x_{4312} \text {. }
\end{aligned}
$$

By the transformation $q=2 \pi j M(m)-t \log M(m)+2 \pi m$, we get

$$
\begin{aligned}
x_{4311} & =\sum_{j=t / 3 \pi+1}^{\infty} \int_{3 \pi j-t \log 3 / 2+2 \pi c_{0}}^{\infty} \frac{1}{2 m(q) M(m(q))^{\sigma}(2 \pi j-t / M(m(q)))} \\
& =0\left(\sum_{j=t / 3 \pi}^{\infty} 1 /(j-t / 3 \pi+1)^{2}\right)=O(1),
\end{aligned}
$$

since $M^{\prime}(m)>0$ and increases and $M^{\prime}(m) / M(m)$ decreases. Similarly $x_{4312}=O(1)$. Therefore $x_{431}=O(1)$. Since $k^{\prime}(p)=1 /(2 \pi j-t / k(p))$, 
we have $X_{432}=O(1), X_{433}=O(1)$. Thus we have proved $x_{43}=O(1)$.

$x_{42}=$

$=\sum_{j=(t / 3 \pi) \log (3 / 2)+1}^{t / 3} \int_{c_{0}}^{\infty} \frac{\sin 2 \pi m}{m} d m \int_{M(m)}^{\infty} \frac{\sin (t \log k-2 \pi j k)}{k^{\sigma}} d k$

$=\sum_{j=(t / 3 \pi) \log (3 / 2)+1}^{t / 3} \int_{3 / 2}^{\infty} \frac{\sin (t \log k-2 \pi j k)}{k^{\sigma}} d k\left(\int_{c_{0}}^{\infty}-\int_{K / 2(1+K / k)}^{\infty}\right) \frac{\sin 2 \pi m}{m} d m$

$=X_{421}-X_{422}$,

where $X_{421}$ is bounded.

$$
\begin{aligned}
x_{422} & =\sum_{j=(t / 3 \pi) \log (3 / 2)+1}^{t / 3 \pi}\left(\int_{3 / 2}^{9}+\int_{9}^{\infty} d k \int_{K / 2(1+K / k)}^{\infty} d m\right. \\
& =x_{4221}+X_{4222}, \\
X_{4222} & =\sum_{j=(t / 3 \pi) \log _{(3 / 2)+1}}^{t / 3 \pi} \int_{18 \pi j-t \log 9}^{\infty} \frac{\sin p d p}{k(p)^{\sigma}(2 \pi j-t / k(p))} \\
& =0\left(\sum_{j=(t / 3 \pi) \log (3 / 2)+1}^{t / 3 \pi} \frac{1}{j}\right)=O(1),
\end{aligned}
$$

and

$$
\begin{aligned}
& x_{4221}=\int_{(t / 3 \pi) \log (3 / 2)+\frac{1}{2}}^{[t / 3 \pi]+\frac{1}{2}}(d j+d J(j)) \int_{3 / 2}^{9} \frac{\sin (t \log k-2 \pi j k)}{k^{\sigma}} d k \\
&=2 \int_{(t / 3 \pi) \log (3 / 2)+\frac{\pi}{2}}^{[t / 3 \pi]+\frac{1}{2}} J(j) d j \int_{3 / 2}^{9} k^{1-\sigma} \cos (t \log k-2 \pi j k) d k \\
& \cdot \int_{K / 2(1+K / k)}^{\infty} \frac{\sin 2 \pi m}{m} d m \\
& \frac{\sin 2 \pi m}{m} d m+o(1) .
\end{aligned}
$$

Writing 


$$
I(k)=\int_{K / 2(1+K / k)}^{\infty} \frac{\sin 2 \pi m}{m} d m,
$$

we get

$$
\begin{aligned}
& X_{4221}=2 \sum_{n=1}^{\infty} \frac{1}{n} \int_{[(t / 3 \pi) \log (3 / 2)]+\frac{1}{2}}^{[t / 3 \pi]+\frac{1}{2}} \sin 2 \pi n j d j \\
& \text { - } \int_{3 / 2}^{9} I(k) k^{1-\sigma} \cos (t \log k-2 \pi j k) d k+o(1) \\
& =\sum_{n=1}^{\infty} \frac{1}{n} \int_{3 / 2}^{9} I(k) k^{1-\sigma} d k \\
& \text { - } \int_{[(t / 3 \pi) \log (3 / 2)]+\frac{1}{2}}^{[t / 3 \pi]+\frac{1}{2}}(\sin (t \log k-2 \pi j(k-n))-\sin (t \log k-2 \pi j(k+n))) d j \\
& =Y_{11}-Y_{12}+O(1), \\
& Y_{11}=\sum_{n=1}^{\infty} \frac{1}{n}\left\{\int_{3 / 2}^{9} I(k) k^{1-\sigma} \sin (t \log k) d k\right. \\
& \text { - } \int_{[(t / 3 \pi) \log (3 / 2)]+\frac{1}{2}}^{[t / 3 \pi]+\frac{1}{2}} \cos (2 \pi j(k-n)) d j+\int_{3 / 2}^{9} I(k) k^{1-\sigma} \cos (t \log k) d k \\
& \left.\int_{[(t / 3 \pi) \log (3 / 2)]+\frac{1}{2}}^{[t / 3 \pi]+\frac{1}{2}} \sin (2 \pi j(k-n)) d j\right\} \\
& =Y_{111}+Y_{112} .
\end{aligned}
$$

We shall write $\theta_{1}=1 /\left([(t / 3 \pi) \log (3 / 2)]+\frac{1}{2}\right), \theta_{2}=1 /\left([t / 3 \pi]+\frac{1}{2}\right)$, and

$$
\begin{aligned}
& y_{111}= \sum_{n=2}^{8} \frac{1}{n}\left(\int_{3 / 2}^{n} d k+\int_{n}^{9} d k\right)+\sum_{n=1,9}+o(1) \\
&= z_{1}+z_{2}+\sum_{n=1,9}+o(1), \\
& Z_{1}=\sum_{n=2}^{8} \frac{1}{n}\left\{\int_{3 / 2}^{n-\theta} 1 k \int_{[(t / 3 \pi) \log (3 / 2)]+\frac{1}{2}}^{[t / 3 \pi]+\frac{1}{2}} d j\right. \\
& \quad+\int_{n-\theta}^{n-\theta} 2 \ln \left(\int_{[(t / 3 \pi) \log (3 / 2)]+\frac{1}{2}}^{1 /(n-k)} d j+\int_{1 /(n-k)}^{[t / 3 \pi]+\frac{1}{2}} d k\right)
\end{aligned}
$$




$$
\begin{aligned}
& =z_{11}+z_{12}+z_{13}+z_{14}, \\
z_{11} & \left.=\sum_{n=2}^{n} \frac{1}{n} \int_{3 / 2}^{n-\theta_{1}} \frac{I(k) k^{1-\sigma_{\sin }(t \log k)}}{n-k} d k \int_{[(t / 3 \pi) \log (3 / 2)]+\frac{\pi}{2}}^{[t / 3 \pi]+\frac{1}{2}} d j\right\} \\
& =z_{111}+z_{112} \cdot
\end{aligned}
$$

In order to estimate $z_{111}$, divide the range of integration into $(3 / 2,7 / 4)$ and $\left(7 / 4, n-\theta_{1}\right)$; then the integrand of the first integral is bounded, but the second is $O\left(1 / \theta_{1} t\right)=O(1)$, using the transformation $p=2 \pi\left([t / 3 \pi]+\frac{1}{2}\right) k-t \log k-2 \pi\left([t / 3 \pi]+\frac{1}{2}\right) n$. About $z_{112}$, in the cases $n \leq 3$, use the transformation $p=t \log k+2 \pi\left([(t / 3 \pi) \log (3 / 2)]+\frac{7}{2}\right)(n-k)$ and, in the cases $n>4$, divide the integration range into $(3 / 2, n-1)$ and $\left(n-1, n-\theta_{1}\right)$; then the integrand of the first integral is bounded and the second integral is $O\left(1 / \theta_{1} t\right)=O(1)$. Thus we have proved that $Z_{11}=O(1) \cdot Z_{13}$ is similarly estimated.

$$
\begin{aligned}
& z_{12}=\sum_{n=2}^{8} \frac{1}{n} \int_{n-\theta_{1}}^{n-\theta_{2}} I(k) k^{1-\sigma} \sin (t \log k) d k \\
& \text { - } \int_{[(t / 3 \pi) \log (3 / 2)]+\frac{1}{2}}^{1 /(n-k)} \sum_{m=0}^{\infty} \frac{(-1)^{m}}{(2 m) !}(2 \pi j(n-k))^{2 m} d j \\
& =\sum_{m=0}^{\infty} \frac{(-1)^{m}}{(2 m) !} \frac{(2 \pi)^{2 m}}{2 m+1} \sum_{n=2}^{8} \frac{1}{n} \int_{n-\theta_{1}}^{n-\theta_{2}} I(k) k^{1-\sigma} \sin (t \log k) \\
& \text { - }\left(\frac{1}{(n-k)^{2 m+1}}-\left([(t / 3 \pi) \log (3 / 2)]+\frac{1}{2}\right)^{2 m+1}\right)(n-k)^{2 m} d k \\
& =O\left(\sum_{m=0}^{\infty} \frac{(2 \pi)^{2 m}}{(2 m+1) !} \sum_{n=2}^{8} \frac{1}{n \theta_{2}}\left(\theta_{1}-\theta_{2}\right)\right)=O(1) \text {. }
\end{aligned}
$$

$Z_{14}$ is also similarly estimated to become bounded. Thus we have proved that $z_{1}$ is bounded. $Z_{2}$ is also, and then $y_{111} \cdot y_{112}$ is derived from 
$Y_{111}$, interchanging sine and cosine, and hence $Y_{112}$ is bounded, and then $Y_{11}$ is so. $Y_{12}$ is easily seen to be bounded. This proves that $X_{4221}$ is bounded. Thus we get that $X_{42}$ is bounded.

Now we shall estimate $X_{41}$.

$$
\begin{aligned}
& x_{41}=\sum_{j=1}^{(t / 3 \pi) \log (3 / 2)} \int_{c_{0}}^{\infty} \frac{\sin 2 \pi m}{m} d m \int_{M(m)}^{\infty} \frac{\sin (t \log k-2 \pi j k)}{k^{\sigma}} d k \\
& =\sum_{j=1}^{(t / 3 \pi) \log (3 / 2)} \int_{3 / 2}^{\infty} \frac{\sin (\log k-2 \pi j k)}{k^{\sigma}} d k \int_{c_{0}}^{k^{\sigma-\varepsilon} / 2\left(1+k^{\sigma-\varepsilon-1}\right)} \frac{\sin 2 \pi m}{m} d m \\
& =\sum_{j=1}^{(t / 3 \pi) \log (3 / 2)}\left[\int_{3 / 2}^{k} d k+\int_{k_{j}}^{\infty} d k\right) \int_{c_{0}}^{k^{\sigma-\varepsilon} / 2\left(1+k^{\sigma-\varepsilon-1}\right)} d n \\
& =x_{411}+x_{412} \text {, } \\
& x_{412}=-\sum_{j=1}^{(t / 3 \pi) \log (3 / 2)} \int_{2 \pi j k_{j}-t \log k_{j}}^{\infty} \frac{\operatorname{sinpdp}}{k(p)^{\sigma}(2 \pi j-t / k(p))} \\
& \cdot \int_{c_{0}}^{k(p)^{\sigma-\varepsilon} / 2\left(1+k(p)^{\sigma-\varepsilon-1}\right)} \frac{\sin 2 \pi m}{m} d m
\end{aligned}
$$

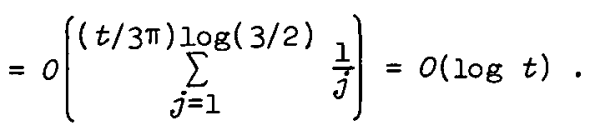

Therefore

$$
\begin{aligned}
V_{124} & =X_{411}+O(\log t) \\
& =\sum_{j=1}^{(t / 3 \pi) \log (3 / 2)} \int_{3 / 2}^{k j} \frac{\sin (t \log k-2 \pi j k)}{k^{\sigma}} d k \int_{c_{0}}^{k^{\sigma-\varepsilon} / 2\left(1+k^{\sigma-\varepsilon-1}\right)} \frac{\sin 2 \pi m}{m} d m \\
& +o(\log t) .
\end{aligned}
$$

Now,

$$
V_{125}=\sum_{j=1}^{\infty} \int_{c_{0}}^{\infty} \frac{\sin 2 \pi m}{m} d m \int_{M(m)}^{\infty}\left(1-\frac{2 m}{k}\right)^{1-\sigma} \frac{\sin (t \log (k-2 m)-2 \pi j k)}{k^{\sigma}} d k
$$




$$
\begin{aligned}
& =\sum_{j=1}^{\infty} \int_{c_{0}}^{\infty} \frac{\sin 2 \pi m}{m} d m \int_{M(m)-2 m}^{\infty}\left(\frac{k}{k+2 m}\right)^{1-\sigma} \frac{\sin (t \log k-2 \pi j k-4 \pi j m)}{(k+2 m)^{\sigma}} d k \\
& =\sum_{j=1}^{\infty} \int_{c_{0}}^{\infty} \frac{\sin 2 \pi m}{m} d m \int_{M(m)-2 m}^{\infty}\{\sin (t \log k-2 \pi j k) \cos 4 \pi j m \\
& -\cos (t \log k-2 \pi j k) \sin 4 \pi j m\} \frac{k^{l-\sigma}}{k+2 m} d k \\
& =x_{51}-x_{52} \text {, } \\
& X_{51}=\sum_{j=1}^{\infty} \int_{c_{0}}^{\infty} \frac{\sin 2 \pi(2 j+1) m-\sin 2 \pi(2 j-1) m}{2 m} d m \int_{M(m)-2 m}^{\infty} \frac{k^{1-\sigma} \sin (t 1 \circ g k-2 \pi j k)}{k+2 m} d k \\
& =X_{511}-X_{512} \text {, } \\
& X_{511}=\sum_{j=1}^{\infty} \frac{\cos 2 \pi(2 j+1) c_{0}}{2 \pi \cdot 2 c_{0}(2 j+1)} \int_{3 / 2-2 c_{0}}^{\infty} \frac{k^{1-\sigma} \sin (t \log k-2 \pi j k)}{k+2 c_{0}} d k \\
& -\sum_{j=1}^{\infty} \int_{c_{0}}^{\infty} \frac{\cos 2 \pi(2 j+1) m}{4 \pi m^{2}(2 j+1)} d m \int_{M(m)-2 m}^{\infty} \frac{k^{1-\sigma} \sin (t \log k-2 \pi j k)}{k+2 m} d k \\
& -\sum_{j=1}^{\infty} \int_{c_{0}}^{\infty} \frac{\cos 2 \pi(2 j+1) m}{4 \pi m(2 j+1)} \frac{(M(m)-2 m)^{1-\sigma}}{M(m)} \\
& \text { - }\left(M^{\prime}(m)-2\right) \sin (t \log (M(m)-2 m)-2 \pi j(M(m)-2 m)) d m \\
& -\sum_{j=1}^{\infty} \int_{c_{0}}^{\infty} \frac{\cos 2 \pi(2 j+1) m}{4 \pi m(2 j+1)} d m \int_{M(m)-2 m}^{\infty} \frac{k^{1-\sigma} \sin (t \log k-2 \pi j k)}{(k+2 m)^{2}} d k \\
& =\frac{1}{4 \pi c_{0}} X_{5111}-\frac{1}{4 \pi}\left(X_{5112}+X_{5113}+X_{5114}\right) \text {. }
\end{aligned}
$$

Let $t^{\prime}=t / 2 \pi\left(3 / 2-2 c_{0}\right)$ and $k_{j}$ be the solution of the equation $2 \pi j k=t \log k$. We use the transformation

$$
p=|2 \pi j k-t \log k|,
$$

where $d p$ vanishes for $k=t / 2 \pi j$. We write 


$$
\begin{aligned}
X_{5111} & \left.=\sum_{j=1}^{t^{\prime}} \frac{\cos 2 \pi(2 j+1) c_{0}}{2 j+1} \iint_{3 / 2-2 c_{0}}^{t / 2 \pi j}+\int_{t / 2 \pi j}^{k j}+\int_{k_{j}}^{\infty}\right) d k \\
& +\sum_{j=t^{\prime}+1}^{\infty} \frac{\cos 2 \pi(2 j+1) c_{0}}{2 j+1} \int_{3 / 2-c_{0}}^{\infty} d k
\end{aligned}
$$

By the transformation ( 7 ), the integral in $Y_{21}$ becomes

$$
\begin{aligned}
\int_{3 / 2-c_{0}}^{t / 2 \pi j} \frac{k^{1-\sigma}}{k+2 c_{0}} \sin (t \log & k-2 \pi j k) d k \\
& =\int_{t \log \left(3 / 2-2 c_{0}\right)-2 \pi j\left(3 / 2-2 c_{0}\right)}^{t \log (t / 2 \pi j e)} \frac{k(p)^{1-\sigma} \sin p d p}{\left(k(p)+2 c_{0}\right)(t / k(p)-2 \pi j)} \\
& =0\left(\int_{t \log (t / 2 \pi j e)-2 \pi}^{t \log (t / 2 \pi j e)} \frac{k(p)^{1-\sigma} d p}{\left(k(p)+2 c_{0}\right)(t / k(p)-2 \pi j)}\right) .
\end{aligned}
$$

For $k=t / 2 \pi j-\theta(0<\theta<t / 2 \pi j)$, in the transformation (7) $p$ is given by

$$
\begin{aligned}
p & =t \log \left(\frac{t}{2 \pi j}-\theta\right)-2 \pi j\left(\frac{t}{2 \pi j}-\theta\right) \\
& =t \log \frac{t}{2 \pi j e}+t \log \left(1-\frac{2 \pi j \theta}{t}\right)+2 \pi j \theta \\
& =t \log \frac{t}{2 \pi j e}-\frac{(2 \pi j \theta)^{2}}{2 t}-\frac{(2 \pi j \theta)^{3}}{3 t^{2}}-\ldots \quad\left(0<\frac{2 \pi j \theta}{t}<1\right) .
\end{aligned}
$$

If we take $\theta=\frac{1}{j} \sqrt{t / \pi}$, then

$$
p=t \log \frac{t}{2 \pi j e}-2 \theta-o(1) .
$$

Therefore, the range $\left(t / 2 j, t / 2 \pi j-\sqrt{t / \pi j^{2}}\right)$ on the $k$ line can be transformed to the interval on the $p$ line, which covers the range of integration of the last integral with respect to $p$. Thus we get 


$$
\begin{aligned}
Y_{2 l} & =0\left(\sum_{j=1}^{t^{\prime}} \frac{1}{j} \int_{t / 2 \pi j-V}^{t / 2 \pi j} \overline{t / \pi j^{2}} \frac{d k}{k^{\sigma}}\right) \\
& =O\left(\sum_{j=1}^{t^{\prime}} \frac{1}{j} \frac{\sqrt{t / \pi j^{2}}}{(t / 2 \pi j)^{\sigma}}\right)=O\left(\sum_{j=1}^{t^{\prime}} \frac{1}{t^{\sigma-\frac{z}{2}} j^{2-\sigma}}\right) \\
& =O(1) .
\end{aligned}
$$

Similarly $Y_{22}, Y_{23}$, and $Y_{24}$ are bounded, and then $X_{5111}=O(1)$.

$$
\begin{aligned}
& x_{5112}=\sum_{j=1}^{t / 2 \pi e}\left\{\int_{c_{0}}^{m} d m\left(\int_{M(m)-2 m}^{t / 2 \pi j}+\int_{t / 2 \pi_{j}}^{k}+\int_{k_{j}}^{\infty}\right) d k\right. \\
& \left.+\int_{m_{0}}^{m_{1}} d m\left[\int_{M(m)-2 m}^{k j}+\int_{k_{j}}^{\infty}\right) d k+\int_{m_{1}}^{\infty} d m \int_{M(m)-2 m}^{\infty} d k\right\} \\
& +\sum_{j=t / 2 \pi e}^{\infty} \int_{c_{0}}^{\infty} d m\left(\int_{M(m)-2 m}^{\max (M(m)-2 m, e)}+\int_{\max (M(m)-2 m, e)}^{\infty}\right) d k \\
& =\sum_{j=1}^{t / 2 \pi e} O\left(\frac{1}{j^{2-\sigma} \sigma_{t} \sigma-\frac{1}{2}}\right)+\sum_{j=1}^{t / 2 \pi e} 0\left(\frac{1}{j}\right)+\sum_{j=t / 2 \pi e}^{\infty} 0\left(\frac{i}{j(j-t / 2 \pi e)}\right) \\
& =O(\log t) \text {. }
\end{aligned}
$$

using an estimate similar to $Y_{21}$ where $m_{0}$ and $m_{1}$ are the solutions of the equation of $m: M(m)-2 m=t / 2 \pi j$ and $M(m)-2 m=k_{j}$, respectively.

Since $k=M(m)$ is the solution of the equation

$$
m=k^{\sigma-\varepsilon} / 2\left(1+k^{\sigma-\varepsilon-1}\right) \text {, }
$$

we have

$$
\begin{aligned}
M^{\prime}(m) & =\frac{d k}{d m}=1 / \frac{d m}{d k} \\
& =\left(\frac{(\sigma-\varepsilon) k^{\sigma-\varepsilon-1}}{2\left(1+k^{\sigma-\varepsilon-1}\right)}-\frac{(\sigma-\varepsilon-1) k^{2(\sigma-\varepsilon-1)}}{2\left(1+k^{\sigma-\varepsilon-1}\right)^{2}}\right)^{-1} \\
& \cong \frac{2}{\sigma-\varepsilon} \frac{1+k^{\sigma-\varepsilon-1}}{k^{\sigma-\varepsilon-1}} \cong \frac{2}{\sigma-\varepsilon} M(m)^{1-\sigma+\varepsilon}
\end{aligned}
$$

and then 


$$
\begin{aligned}
& X_{5113}= \\
& =\sum_{j=1}^{\infty} \frac{1}{4 \pi(2 j+1)} \int_{c_{0}}^{\infty} \frac{(M(m)-2 m)^{1-\sigma}(M(m)-2)}{m M(m)} \\
& \text { - }\{\sin t(\log (M(m)-2 m)-2 \pi m-2 \pi j M(m))+\sin (t \log (M(m)-2 m)+2 \pi m-2 \pi j(M(m)-4 m))\} d m \\
& =\sum_{j=1}^{t / 2 \pi c_{0}^{1 /(\sigma-\varepsilon)}}\left[\int_{c_{0}}^{(t / 2 \pi j)^{\sigma-\varepsilon}}+\int_{(t / 2 \pi j)^{\sigma-\varepsilon}}^{\infty}\right] d m+\sum_{j=t / 2 \pi c_{0}^{1 /(\sigma-\varepsilon)}}^{\infty} \int_{c_{0}}^{\infty} d m \\
& =O(1) \text {. } \\
& x_{5114} \text { is also of the same order as } x_{5113} \text {, and then } \\
& X_{511}=O(\log t) \text {. }
\end{aligned}
$$

$x_{512}$ is similarly estimated, and then $x_{51}$ is also of order $O(\log t)$, and $X_{52}$ is also. Therefore

$$
V_{125}=O(\log t)
$$

$V_{126}$ is quite similar to $V_{125}$, so that

$$
\begin{aligned}
V_{12} & =\frac{1}{2} V_{124}+O(\log t) \\
& =\frac{1}{2} \sum_{j=1}^{(t / 3 \pi) \log (3 / 2)} \int_{3 / 2}^{k} \frac{\sin (\operatorname{tog} k-2 \pi j k)}{k^{\sigma}} d k \int_{c_{0}}^{k^{\sigma-\varepsilon / 2\left(1+k^{\sigma-\varepsilon-1}\right)}} \frac{\sin 2 \pi m}{m} d m \\
& +O(\log t) .
\end{aligned}
$$

Combining with the estimation of $\$ 7.2 .1$ we get

$$
\begin{aligned}
& V_{1}=V_{11}+V_{12} \\
&=\frac{3}{2} \sum_{j=1}^{t / 3 \pi} \int_{3 / 2}^{k} \frac{\sin (t \log k-2 \pi j k)}{k^{\sigma}} d k \int_{0}^{k^{\sigma-\varepsilon} / 2\left(1+k^{\sigma-\varepsilon-1}\right)} \frac{\sin 2 \pi m}{m} d m \\
&+O(\log t) .
\end{aligned}
$$

Denoting the inner integral of $V_{2}$ by $g(k)$, we have 


$$
\begin{aligned}
V_{2}=\frac{1}{t} & \sum_{j=1}^{\infty}\left\{\left[\frac{\sin (t \log k) \sin 2 \pi j k}{2 \pi j k^{\sigma}} g(k)\right]_{k=3 / 2}^{\infty}\right. \\
& \left.-\int_{3 / 2}^{\infty} \frac{t \cos (t \log k) \sin 2 \pi j k}{2 \pi j k^{1+\sigma}} g(k) d k+\int_{3 / 2}^{\infty} \frac{\sin (t \log k) \sin 2 \pi j k}{2 \pi j}\left(\frac{g(k)}{k^{\sigma}}\right)^{\prime} d k\right\} \\
= & O(1) .
\end{aligned}
$$

We easily see that $k_{j}$, the upper limit of the outer integral in $V_{1}$, can be replaced by $t / j \pi$. Therefore, combining with the estimation of $T_{111}$ and $T_{12}=O(1)$, we get

$$
\begin{aligned}
T_{1} & =\sum_{j=1}^{t / 3 \pi} \int_{3 / 2}^{t / j \pi} \frac{\sin (t \log k-2 \pi j k)}{k^{\sigma}} d k \int_{0}^{k^{\sigma-\varepsilon} / 2\left(1+k^{\sigma-\varepsilon-1}\right)} \frac{\sin 2 \pi m}{m} d m+O(\log t) \\
& =\sum_{j=1}^{t / 3 \pi}\left(\int_{3 / 2}^{t / 2 \pi j}+\int_{t / 2 \pi j}^{t / \pi j}\right) d k\left(\int_{0}^{\infty}-\int_{k^{\sigma-\varepsilon} / 2\left(1+k^{\sigma-\varepsilon-1}\right)}^{\infty}\right) d m+o(\log t) \\
& =T_{11}^{\prime}+T_{12}^{\prime}-\left(T_{13}^{\prime}+T_{14}^{\prime}\right)+o(\log t) .
\end{aligned}
$$

Now

$$
\begin{aligned}
T_{13}^{\prime} & =\sum_{j=1}^{t / 3 \pi} \int_{3 / 2}^{t / 2 \pi j} \frac{\sin (t \log k) \cos 2 \pi j k-\cos (t \log k) \sin 2 \pi j k}{k^{\sigma}} h(k) d k \\
& =T_{131}^{\prime}-T_{132}^{\prime},
\end{aligned}
$$

where

$$
h(k)=\int_{k^{\sigma-\varepsilon} / 2\left(1+k^{\sigma-\varepsilon-1}\right)}^{\infty} \frac{\sin 2 \pi m}{m} d m .
$$

We write

$$
\begin{aligned}
T_{131}^{\prime} & =\int_{\frac{1}{2}}^{[t / 3 \pi]+\frac{1}{2}}(d j+d J(j)) \int_{3 / 2}^{t / 2 \pi j} \frac{\sin (t \log k) \cos 2 \pi j k}{k^{\sigma}} h(k) d k \\
& =U_{1}^{\prime}+U_{2}^{\prime},
\end{aligned}
$$

where 


$$
\begin{aligned}
U_{1}^{\prime} & =\int_{3 / 2}^{t / \pi} \frac{\sin (t \log k)}{k^{\sigma}} h(k) d k \int_{\frac{1}{2}}^{t / 2 \pi k} \cos 2 \pi j k d j+O(1) \\
& =0\left(\int_{3 / 2}^{t / \pi} \frac{d k}{k^{2 \sigma-\varepsilon_{k}}}\right)+o(1)=O(1),
\end{aligned}
$$

and using the transformation $t \log (t / 2 \pi j)=t \log \left(t / 2 \pi j^{\prime}\right)+\pi$,

$$
\begin{aligned}
U_{2}^{\prime}= & \int_{\frac{1}{2}}^{[t / 3 \pi]+\frac{1}{2}} J(j) \frac{t}{2 \pi j^{2}} \frac{\sin (t \log (t / 2 \pi j)) \cos t}{(t / 2 \pi j)^{\sigma}} h\left(\frac{t}{2 \pi j}\right) d j \\
& +2 \pi \int_{\frac{1}{2}}^{[t / 3 \pi]+\frac{1}{2}} J(j) d j \int_{3 / 2}^{t / 2 \pi j} k^{1-\sigma} \sin (t \log k) \sin 2 \pi j k \cdot h(k) d k \\
= & O(1)+2 \pi \int_{3 / 2}^{t / \pi} k^{1-\sigma} h(k) \sin (t \log k) d k \int_{\frac{1}{2}}^{t / 2 \pi k} \sin 2 \pi j k J(j) d j+O(1) \\
= & O(1)+\int_{3 / 2}^{t / \pi} \frac{h(k) \sin (t \log k)}{k^{\sigma}} d k\left\{-\cos t J\left(\frac{t}{2 \pi k}\right)+\int_{\frac{1}{2}}^{t / 2 \pi k} \cos 2 \pi j k d J(j)\right\} \\
= & O(1)+o\left(\int_{3 / 2}^{t / \pi} \frac{\log (t / 2 \pi k)}{k^{2 \sigma-\varepsilon}} d k\right)=O(\log t) .
\end{aligned}
$$

Thus we have proved that $T_{131}^{\prime}=O(1)$. Similarly $T_{132}^{\prime}$ is bounded, and then $T_{13}^{\prime}$ is also. Since $T_{14}^{\prime}$ is also bounded by a similar estimate, we get

$$
T_{1}=\frac{\pi}{2} \sum_{j=1}^{t / 3 \pi} \int_{3 / 2}^{t / j \pi} \frac{\sin (t \log k-2 \pi j k)}{k^{\sigma}} d k+o(\log t)
$$

\section{Estimation of the remaining terms}

8.1. Estimation of $T_{2}$.

$T_{2}=$

$=\sum_{k=2}^{\infty} \frac{1}{k^{\sigma}} \int_{0}^{t \log (1+K / k)} \cos (t \log k+\omega)$

- $\left(e^{-(1-\sigma) w / t} \int_{k\left(1-e^{-\omega / t}\right) / 2}^{\infty} \frac{\sin 2 \pi m}{m} d m-e^{(1-\sigma) w / t} \int_{k\left(e^{w / t}-1\right) / 2}^{\infty} \frac{\sin 2 \pi m}{m} d m\right) d \omega$ 


$$
\begin{aligned}
& =\sum_{k=2}^{\infty} \frac{1}{k^{\sigma}}\left\{\int_{0}^{t \log (1+K / k)} \cos (t \log k+\omega)\left(e^{-(1-\sigma) w / t}-e^{(1-\sigma) w / t}\right) d \omega\right. \\
& \cdot \int_{k\left(1-e^{-w / t}\right) / 2}^{\infty} \frac{\sin 2 \pi m}{m} d m \\
& \left.-\int_{0}^{t \log (1+K / k)} \cos (t \log k+\omega) e^{(1-\sigma) w / t} d \omega \int_{k\left(1-e^{-\omega / t}\right) / 2}^{k\left(e^{w / t}-1\right) / 2} \frac{\sin 2 \pi m}{m} d m\right\} \\
& =-T_{21}-T_{22} \text {, } \\
& T_{21}=\sum_{n=1}^{\infty} \frac{2(1-\sigma)^{2 n-1}}{(2 n-1)} \sum_{k=2}^{\infty} \frac{1}{k^{\sigma} t^{2 n-1}} \int_{0}^{t \log (1+K / k)} \cos (t \log k+w) w^{2 n-1} d w \\
& \int_{k\left(1-e^{-w / t}\right) / 2}^{\infty} \frac{\sin 2 \pi m}{m} d m
\end{aligned}
$$

by using the expansion formula for $e^{x}$, and denoting by $I(k, w)$ the inner integral in the last formula, we get

$$
\begin{aligned}
& \int_{0}^{t \log (I+K / k)} \cos (t \log k+w) w^{2 n-1} I(k, w) d w \\
&=\left[\sin (t \log k+w) w^{2 n-I} I(k, w)\right]_{w=0}^{t \log (1+K / k)} \\
& \quad-\int_{0}^{t \log (1+K / k)} \sin (t \log k+w) \\
&\left\{(2 n-1) w^{2 n-2} I(k, w)+w^{2 n-1} \frac{k \sin \pi k\left(1-e^{-w / t}\right)}{k t\left(1-e^{-w / t}\right)} e^{-w / t}\right\} d w,
\end{aligned}
$$

and then $T_{21}=O(1) \cdot T_{22}$ is also bounded by the same method. Thus we have proved that $T_{2}=O(1)$.

8.3. Estimation of $T_{3}$. Similarly as the estimation of $T_{2}$, we get 


$$
\begin{aligned}
& T_{3}=\sum_{k=2}^{\infty} \frac{1}{k^{\sigma}} \int_{t \log (1+K / k)}^{-t \log (1-K / k)} \cos (t \log k-w) e^{-(1-\sigma) w / t} d v \\
& \int_{k\left(1-e^{-\omega / t}\right) / 2}^{\infty} \frac{\sin 2 \pi m}{m} d n \\
& =O(1) \text {. } \\
& \text { 8.3. Estimation of } Q_{222}, Q_{223} \text {, and } Q_{224} \text {. } \\
& Q_{222}=\sum_{k=2}^{\infty} \frac{t}{2 \pi k} \int_{k}^{k-1} \frac{\cos (t \log (k-u))}{(k-u)^{\sigma}} d u \int_{u / 2}^{\infty} \frac{\sin 2 \pi m}{m} d u \\
& =\sum_{k=2}^{\infty} \frac{1}{2 \pi k} \int_{0}^{t \log (k-K)} e^{(1-\sigma) v / t} \cos v d v \int_{\left(k-e^{v / t}\right) / 2}^{\infty} \frac{\sin 2 \pi m}{m} d m \\
& =\sum_{k=2}^{\infty} \frac{1}{2 \pi k} \int_{0}^{t \log (k-K)} e^{(1-\sigma) v / t} \cos v d v \\
& \cdot\left\{\frac{\cos \left(k-e^{v / t}\right)}{\left(k-e^{v / t}\right)}-\int_{\left(k-e^{v / t}\right) / 2}^{\infty} \frac{\cos 2 \pi m}{2 \pi m^{2}} d m\right\} \\
& =\frac{1}{2 \pi^{2}} R_{1}^{\prime}-\frac{1}{4 \pi^{2}} R_{2}^{\prime},
\end{aligned}
$$

where

$$
\begin{aligned}
R_{1}^{\prime} & =\sum_{k=2}^{\infty} \frac{(-1)^{k}}{k} \int_{0}^{t \log (k-K)} \frac{e^{(1-\sigma) v / t}}{k-e^{v / t}} \cos \left(\pi e^{v / t}-v\right) d v+O(1) \\
& =\sum_{k=2}^{t / \pi}+\sum_{k=t / \pi+1}^{\infty}+O(1) \\
& =R_{11}^{\prime}+R_{12}^{\prime}+O(1) .
\end{aligned}
$$

We use the transformation $p=v-\pi e^{v / t}$, where $d p=\left(1-\frac{\pi}{t} e^{v / t}\right) d v$, vanishing at $v=t \log \frac{t}{\pi}$. For $v=t \log (t / \pi)$, we have $p=t \log (t / \pi e)$ and for $v=t \log (t / \pi)-\theta$,

$$
\begin{aligned}
p & =t \log (t / \pi e)-\theta-t\left(1-e^{-\theta / t}\right) \\
& \cong p_{0}-\frac{1}{2} \theta^{2} / t .
\end{aligned}
$$

If we take $\theta=\sqrt{4 \pi t}$, then we see that $p$ changes over an interval of 
length greater than $2 \pi$ when $v$ changes from $t \log (t / \pi)-\sqrt{4 \pi t}$ to $t \log (t / \pi)$. Therefore

$$
\begin{aligned}
R_{12}^{\prime} & =0\left(\sum_{k=t / \pi+1}^{\infty} \frac{1}{k} \int_{t \log (t / \pi)-\sqrt{4 \pi t}}^{t \log (t / \pi)} \frac{e^{(1-\sigma) v / t}}{k-e^{v / t}} d v\right) \\
& =0\left(t^{1-\sigma+\frac{1}{2}} \sum_{k=t / \pi+1}^{\infty} \frac{1}{k(k-t / \pi)}\right)=O(1) .
\end{aligned}
$$

Similarly,

$$
R_{I I}^{\prime}=0\left(\sum_{k=2}^{t / \pi} \frac{1}{k} \frac{k^{1-\sigma}}{k^{\sigma-\varepsilon}} \frac{t}{t-k \pi+K \pi}\right)=O(1) .
$$

Thus $R_{1}^{\prime}=O(1)$.

$$
\begin{aligned}
R_{2}^{\prime} & =\sum_{k=2}^{\infty} \frac{1}{k} \int_{0}^{t \log (k-K)} e^{(1-\sigma) v / t} \cos v d v \int_{\left(k-e^{v / t}\right) / 2}^{\infty} \frac{\cos 2 \pi m}{m^{2}} d m \\
& =\sum_{k=2}^{\infty} \frac{1}{k}\left\{\int_{K}^{k / 2-\frac{1}{2}} d m \int_{t \log (k-2 m)}^{t \log (k-K)} d v+\int_{k / 2-\frac{1}{2}}^{\infty} d m \int_{0}^{t \log (k-K)} d v\right\} \\
& =o(1) .
\end{aligned}
$$

Thus we have proved that $Q_{222}$ is bounded. Similarly $Q_{223}$ and $Q_{224}$ are bounded.

Collecting the above estimates we get

$$
I \zeta(s)=-\sum_{j=1}^{t / 3 \pi} \int_{3 / 2}^{t / \pi j} \frac{\sin (t \log k-2 \pi j k)}{k^{\sigma}} d k+o(\log t) .
$$

For the real part of $\zeta(s)$, we get a corresponding formula where sine is replaced by cosine. Therefore

$$
\zeta(s)=-\sum_{j=1}^{t / 3 \pi} \int_{3 / 2}^{t / \pi j} e^{i(t \log k-2 \pi j k)} \frac{d k}{k^{\sigma}}+o(\log t) .
$$

Thus Theorem 1 is proved. 


$$
\begin{aligned}
9 . & \text { Proof of Theorem } 2 \\
G & =\sum_{j=1}^{t / 3 \pi} \int_{3 / 2}^{t / \pi j} \frac{\sin (t \log k-2 \pi j k)}{k^{\sigma}} d k \\
& =\int_{\frac{3}{2}}^{[t / 3 \pi]+\frac{2}{2}}(d j+d J(j)) \int_{3 / 2}^{t / \pi j} \frac{\sin (t] \circ g k-2 \pi j k)}{k^{\sigma}} d k \\
& =G_{1}+G_{2} .
\end{aligned}
$$

By change of order of integration, we can easily see that $G_{1}=O(1)$.

$$
\begin{aligned}
G_{2} & =\int_{\frac{\frac{1}{2}}{2}}^{[t / 3 \pi]+\frac{1}{2}} d J(j) \int_{3 / 2}^{t / 2 \pi j} \frac{\sin (t \log k-2 \pi j k)}{k^{\sigma}} d k+\int_{\frac{2}{2}}^{[t / 3 \pi]+\frac{1}{2}} d J(j) \int_{t / 2 \pi j}^{t / \pi j} d k \\
& =G_{21}+G_{22} .
\end{aligned}
$$

By integration by parts,

$$
\begin{aligned}
G_{21}= & \int_{\frac{1}{2}}^{[t / 3 \pi]+\frac{3}{2}} J(j) \\
& \frac{t}{2 \pi j^{2}} \frac{\sin (t \log (t / 2 \pi j e)]}{(t / 2 \pi j)^{\sigma}} d j \\
& \quad+2 \pi \int_{\frac{1}{2}}^{[t / 3 \pi]+\frac{1}{2}} J(j) d j \int_{3 / 2}^{t / 2 \pi j} k^{1-\sigma} \cos (t \log k-2 \pi j k) d k \\
= & \frac{1}{(2 \pi)^{1-\sigma}} G_{211}+2 \pi G_{212},
\end{aligned}
$$

where

$$
\begin{aligned}
G_{211} & =t^{1-\sigma} \int_{\frac{1}{2}}^{[t / 3 \pi]+\frac{1}{2}} \frac{J(j)}{j^{2-\sigma} \sin (t \log (t / 2 \pi j e)) d j} \\
& =t^{1-\sigma} \sum_{n=1}^{t / 3 \pi} \int_{n}^{n+1} d j+o(1) \\
& =t^{1-\sigma} \sum_{n=1}^{t / 3 \pi} \int_{t \log (t / 2 \pi(n+1) e)}^{t \log (t / 2 \pi n e)} \frac{e^{(1-\sigma) p / t} \sin p}{(t / 2 \pi e)^{2-\sigma}} d p \\
& =o\left(\frac{1}{t} \sum_{t=1}^{t / 3 \pi} \frac{1}{n^{1-\sigma}}\right)=o(1),
\end{aligned}
$$




$$
\begin{aligned}
G_{212}= & \int_{3 / 2}^{t / \pi} k^{1-\sigma} \cos (t \log k) d k \int_{\frac{1}{2}}^{t / 2 \pi k} \cos (2 \pi j k) J(j) d j \\
& \quad+(\text { the term where the cosine is replaced by sine })+O(1) \\
= & H_{1}+H_{2}+O(1) .
\end{aligned}
$$

Using integration by parts,

$$
\begin{aligned}
& H_{1}=\int_{3 / 2}^{t / \pi} k^{I-\sigma} \cos (t \log k)\left\{\left[\frac{\sin 2 \pi j k}{2 \pi k} J(j)\right]_{j=\frac{1}{2}}^{t / 2 \pi k}-\frac{1}{2 \pi k} \int_{\frac{1}{2}}^{t / 2 \pi k} \sin 2 \pi j k d J(j)\right\} \\
&=\frac{1}{2 \pi} H_{11}-\frac{1}{2 \pi} H_{12}, \\
& H_{11}=\sin t \int_{3 / 2}^{t / \pi} \frac{\cos (t \log k)}{k^{\sigma}} J\left(\frac{t}{2 \pi k}\right) d k \\
&=\sin t \sum_{n=1}^{t / \pi} \int_{t / 2 \pi(n+1)}^{t / 2 \pi n}+O(1) \\
&=\sin t \sum_{n=1}^{t / \pi}\left(\int_{t / 2 \pi(n+1)}^{\theta}-\int_{\theta^{\prime}}^{t / 2 \pi n}\right) \frac{\cos (t \log k)}{k^{\sigma}} d k
\end{aligned}
$$

where $t / 2 \pi(n+1)<\theta<t / 2 \pi\left(n+\frac{1}{2}\right)<\theta^{\prime}<t / 2 \pi n$. Using the transformation $p=t \log k, d p=(t / k) d k$, we get

$$
H_{11}=0\left(\sum_{n=1}^{t / \pi} \frac{1}{t}\left[\frac{t}{n}\right)^{1-\sigma}\right)=O(1) .
$$

Further

$$
H_{12}=\int_{3 / 2}^{t / \pi} \frac{\cos (t \log k)}{k^{\sigma}} \bar{D}_{[t / 2 \pi k]}(k) d k
$$

where $\bar{D}_{n}(k)$ denotes the $n$th conjugate Dirichlet kernel at the point $k$, and then

$$
\begin{aligned}
H_{12} & =\sum_{n=1}^{\sqrt{t / 2 \pi}} \int_{t / 2 \pi(n+1)}^{t / 2 \pi n}+\int_{t / 2 \pi}^{t / \pi}+\sum_{m=1}^{\sqrt{t}-1} \int_{m}^{m+1}+\int_{[\sqrt{t}]}^{t / 2 \pi([\sqrt{t / 2 \pi}]+1)} \\
& =H_{121}+H_{122}+H_{123}+H_{124},
\end{aligned}
$$

where 


$$
\begin{aligned}
& H_{121}=\sum_{n=1}^{\sqrt{t / 2 \pi}} \int_{t / 2 \pi(n+1)}^{t / 2 \pi n} \frac{\cos (t \log k)}{k^{\sigma}} \bar{D}_{n}(2 \pi k) d k \\
& =\sum_{n=1}^{\sqrt{t / 2 \pi}}\left(\sum_{l=1}^{n} \int_{t / 2 \pi(n+1)}^{t / 2 \pi n} \frac{\sin (t \log k-2 \pi l k)}{k^{\sigma}} d k\right)+o(1) \\
& =0\left(\sum_{n=1}^{\sqrt{t / 2 \pi}}\left(\sum_{l=1}^{n-1}\left(\frac{t}{n}\right)^{1-\sigma} \frac{1}{t-l t / n}+\left(\frac{n}{t}\right)^{\sigma} \frac{\sqrt{t}}{n}\right)\right)+O(1) \\
& =o\left(t^{(1-\sigma) / 2} \log t\right) \text {, } \\
& H_{123}=0\left(\sum_{m=1}^{\sqrt{t-1}} \frac{1}{m^{\sigma}} \log \frac{t}{2 \pi m}\right)=O\left(t^{(1-\sigma) / 2} \log t\right) \text {, }
\end{aligned}
$$

and $H_{122}$ and $H_{124}$ are bounded. Thus we have proved that $H_{1}$ is of order $O\left(t^{(1-\sigma) / 2} \log t\right) \cdot H_{2}$ is also and then $G_{21}$ is of the same order. $G_{22}$ can be estimated similarly and more easily. Therefore we have proved that $G=o\left(t^{(1-\sigma) / 2} \log t\right)$, which proves Theorem 2 .

\section{Appendix}

It is sufficient to prove that

$$
I_{1}=\int_{\frac{1}{2}}^{M} \frac{J(m)}{m} d m \int_{L}^{\infty} \frac{\cos (t \log u) \cos 2 \pi m u}{u^{\sigma}} d u \rightarrow 0 \text { as } L \rightarrow \infty \text {, for all } M>0 \text {, }
$$

and

$$
I_{2}=\int_{1}^{\infty} \frac{\cos (t \log u)}{u^{\sigma}} d u \int_{M}^{\infty} J(m) \cos 2 \pi m u \frac{d m}{m} \rightarrow 0 \text { as } M \rightarrow \infty
$$

Now, suppose $L>t \log t$,

$$
\begin{aligned}
I_{1} & =\frac{1}{2} \int_{\frac{2}{2}}^{M} \frac{J(m)}{m} d m \int_{L}^{\infty} \frac{\cos (2 \pi m u-t \log u)+\cos (2 \pi m u+t \log u)}{u^{\sigma}} d u \\
& =\frac{1}{2}\left(I_{11}+I_{12}\right) .
\end{aligned}
$$

By the transformation $v=2 \pi m u-t \log u$ and denoting by $u(v)$ the solution for $u$ of the above equation for fixed $v$, 


$$
\begin{aligned}
I_{11} & =\int_{\frac{1}{2}}^{M} \frac{J(m)}{m} d m \int_{2 \pi m L-t \log L}^{\infty} \frac{\cos v d v}{u(v)^{\sigma}(2 \pi m-t / u(v))} \\
& =-0\left(\frac{1}{L^{\sigma}} \int_{\frac{1}{2}}^{\infty} \frac{d m}{m^{2}}\right)=o(1) \text { as } L \rightarrow \infty .
\end{aligned}
$$

By the transformation $v=2 \pi m u+t \log u$, we can prove similarly that $I_{12}=o(1)$.

$\int_{M}^{\infty} J(m) \cos 2 \pi m u \frac{d m}{m}=\left[\frac{1}{m} \int_{M}^{m} J(n) \cos 2 \pi n u d n\right]_{m=\infty}-\int_{M}^{\infty} \frac{d n}{m^{2}} \int_{M}^{m} J(n) \cos 2 \pi n u d n$

where

$$
\begin{aligned}
\int_{M}^{m} J(n) \cos & 2 \pi n u d n \\
& =\sum_{k=1}^{\infty} \frac{1}{\pi k} \int_{M}^{m} \sin 2 \pi k n \cos 2 \pi n u d n \\
& =\sum_{k=1}^{\infty} \frac{1}{2 \pi k} \int_{M}^{m}\{\sin 2 \pi(k+u) n+\sin 2 \pi(k-u) n\} d n \\
& =\sum_{k=1}^{\infty} \frac{1}{2 \pi k}\left\{\frac{\cos 2 \pi(k+u) M-\cos 2 \pi(k+u) m}{2 \pi(k+u)}+\frac{\cos 2 \pi(k-u) M-\cos 2 \pi(k-u) m}{2 \pi(k-u)}\right\},
\end{aligned}
$$

and then

$$
\begin{gathered}
\lim _{m \rightarrow \infty} \frac{1}{m} \int_{M}^{m} J(n) \cos 2 \pi n u d n=o(1), \\
\int_{M}^{\infty} J(m) \cos 2 \pi m u \frac{d m}{m}=-\sum_{k=1}^{\infty} \frac{1}{(2 \pi)^{2} M k} \frac{\cos 2 \pi(k+u) M}{k+u} \\
+\sum_{k=1}^{\infty} \frac{1}{(2 \pi)^{2} M k}\left(\frac{1}{k+u} \int_{M}^{\infty} \frac{\cos 2 \pi(k+u) m}{m^{2}} d n+\frac{1}{k+u} \int_{M}^{\infty} \frac{\cos 2 \pi(k-u) m-\cos 2 \pi(k-u) M}{m^{2}} d m\right),
\end{gathered}
$$

and 


$$
\begin{aligned}
& I_{2}=\int_{1}^{\infty} \frac{\cos (t \log u)}{u^{\sigma}}\left(\sum_{k=1}^{\infty} \frac{1}{(2 \pi)^{2}(k-u) k}\right. \\
& \left.\cdot \int_{M}^{\infty} \frac{\cos 2 \pi(k-u) m-\cos 2 \pi(k-u) M}{m^{2}} d m\right) d u+o(1) \\
& \left.=\iint_{1}^{3 / 2}+\sum_{j=2}^{\infty} \int_{j-\frac{1}{2}}^{j+\frac{1}{2}}\right) \frac{\cos (t \log u)}{u^{\sigma}} d u\left(\sum_{k=1}^{j-1}+\sum_{k=j}+\sum_{k=j+1}^{\infty}\right)+o(1) \\
& =\frac{1}{(2 \pi)^{2}} \int_{1}^{3 / 2} \frac{\cos (t \log u)}{(u-1) u^{\sigma}} d u \int_{M}^{\infty} \frac{\cos 2 \pi(j-u) m-\cos 2 \pi(j-u) M}{m^{2}} d m \\
& +\frac{1}{(2 \pi)^{2}} \sum_{j=2}^{\infty} \int_{j-\frac{1}{2}}^{j+\frac{1}{2}} \frac{\cos (t \log u)}{j u^{\sigma}(j-u)} d u \int_{M}^{\infty} \frac{\cos 2 \pi(j-u) m-\cos 2 \pi(j-u) M}{m^{2}} d m+o(1) \\
& =o\left(\int_{1}^{1+1 / M} \frac{d u}{d \sigma}\left(\int_{(u-1) M}^{1} \frac{d m}{m}+\int_{1}^{\infty} \frac{d m}{m^{2}}\right)+\frac{1}{M} \int_{1+1 / M}^{3 / 2} \frac{d u}{(u-1) u^{\sigma}}\right) \\
& +\frac{1}{(2 \pi)^{2}} \sum_{j=2}^{\infty} \frac{1}{j} \int_{0}^{\frac{\pi}{2}}\left(\frac{\cos (t \log (j+u))}{(j+u)^{\sigma}}-\frac{\cos (t \log (j-u))}{(j-u)^{\sigma}}\right) d u \\
& \text { - } \int_{(j-u) M}^{\infty} \frac{\cos 2 \pi m-\cos 2 \pi(j-u) M}{m^{2}} d m+o(1) \\
& =o(1) \text {. }
\end{aligned}
$$

Thus we have proved that the order of integration of $Q_{2}$ can be interchanged, that is,

$$
Q_{2}=t \int_{1}^{\infty} \frac{\cos (t \log u)}{u^{\sigma}} d u \int_{\frac{1}{2}}^{\infty} \frac{J(m)}{m} \cos 2 \pi m u d m .
$$

\section{References}

[1] H.M. Edwards, Riemonn's zeta function (Academic Press, New York, London, 1974).

[2] G.H. Hardy and J.E. Littlewood, "The approximate functional equation in the theory of the zeta-function, with applications to the divisor-problems of Dirichlet and Piltz", Proc. London Math. Soc. (2) $21(1922), 39-74$. 
[3] E.C. Titchmarsh, The theory of the Riemonn zeta-function (Clarendon Press, Oxford, 1951).

Department of Mathematics,

Institute of Advanced Studies,

Australian National University,

Canberra, ACT. 